\title{
ALGEBRAS STABLY EQUIVALENT TO SELFINJECTIVE ALGEBRAS WHOSE AUSLANDER-REITEN QUIVERS CONSIST ONLY OF GENERALIZED STANDARD COMPONENTS
}

\author{
by ZYGMUNT POGORZAŁY'
}

(Received 27 November, 1995; revised 10 February, 1997)

0. Introduction. Throughout the paper $K$ denotes a fixed algebraically closed field. All algebras considered are finite-dimensional associative $K$-algebras with a unit element. Moreover, they are assumed to be basic and connected. For an algebra $A$ we denote by $\bmod (A)$ the category of all finitely generated right $A$-modules, and $\bmod (A)$ denotes the stable category of $\bmod (A)$, i.e. $\bmod (A) / \mathscr{P}$ where $\mathscr{P}$ is the two-sided ideal in $\bmod (A)$ of all morphisms that factorize through projective $A$-modules. Two algebras $A$ and $B$ are said to be stably equivalent if the stable categories $\underline{\bmod }(A)$ and $\underline{\bmod }(B)$ are equivalent. The study of stable equivalences of algebras has its sources in modular representation theory of finite groups. It is of importance in this theory whether two stably equivalent algebras have the same number of pairwise non-isomorphic nonprojective simple modules. Another motivation for studying stable equivalences appears in the following context. If $E$ is a $K$-algebra of finite global dimension then its derived category $\mathbf{D}^{b}(E)$ is equivalent to the stable category $\bmod (\hat{E})$ of the repetitive category $\hat{E}$ of $E[15]$. Thus the problem of a classification of derived equivalent algebras leads in many cases to a classification of stably equivalent selfinjective algebras.

We are interested in algebras which are stably equivalent to representation-infinite selfinjective algebras whose Auslander-Reiten quivers consist only of generalized standard components in the sense of Skowronski [30]. It was announced in [34] that representation-infinite selfinjective algebras, whose Auslander-Reiten quivers consist only of generalized standard components, are standard algebras of polynomial growth (for the needed definitions see Section 1). Since there is given a classification of such algebras in [32] we can use it to describe algebras stably equivalent to representation-infinite selfinjective algebras whose Auslander-Reiten quivers consist only of generalized standard components. The following theorem is the main result of the paper.

THEOREM. Let $B$ be a selfinjective representation-infinite algebra in which all components of the Auslander-Reiten quiver $\Gamma_{B}$ are generalized standard. If $C$ is an algebra which is stably equivalent to $B$ then $C$ is a standard selfinjective algebra of polynomial growth. Moreover $B$ and $C$ have the same number of pairwise non-isomorphic simple modules.

Recall that the algebras stably equivalent to representation-finite selfinjective algebras were classified by Riedtmann in $[24,25,26,9]$. Algebras stably equivalent to tame trivial extensions were described in $[\mathbf{2 0 , 2 3 , 2 2 ]}$. In both cases there was linked a tilting-cotilting equivalence of some factor algebras to any stable equivalence. In our case the situation is a little bit different. Any stable equivalence of $B$ and $C$ can be lifted to a stable equivalence of Galois coverings $\tilde{B}, \tilde{C}$ of $B$ and $C$, respectively. Furthermore the

\footnotetext{
' Supported by Polish Scientific Grant KBN No 590/P03/95/08.
} 
lifted stable equivalence of $\tilde{B}$ and $\tilde{C}$ is induced by a tilting-cotilting equivalence of some subcategories in $\tilde{B}$ and $\tilde{C}$.

\section{Preliminaries.}

1.1. Let $K[X]$ be the polynomial algebra in one variable. Following Drozd [12] an algebra $A$ is called tame if, for any dimension $d$, there is a finite number of $K[X]$ - $A$-bimodules $Q_{i}, \quad 1 \leq i \leq n_{d}$, which are finitely generated and free as right $K[X]$-modules, and satisfy the following condition: all but a finite number of isomorphism classes of indecomposable $A$-modules of dimension $d$ are of the form $K[X] /(X-\lambda) \otimes_{K|X|} Q_{i}$ for some $\lambda \in K$ and some $i, 1 \leq i \leq n_{d}$.

Denote by $\mu_{A}(d)$ the least number of bimodules $Q_{i}$ satisfying the above condition for $d$. Then $A$ is said to be of polynomial growth [31] if there is a natural number $m$ such that $\mu_{A}(d) \leq d^{\prime \prime}$.

1.2. Let $R$ be a locally bounded $K$-category $[\mathbf{8}]$. We denote by $\bmod (R)$ the category of all finite-dimensional contravariant functors from $R$ to the category of $K$-vector spaces. For a group $G$ of $K$-linear automorphisms of $R$ acting freely on the objects of $R, R / G$ denotes the quotient category [14] whose objects are the $G$-orbits of the objects of $R$. There is a Galois covering functor $F: R \rightarrow R / G$ which assigns to each object $x$ its $G$-orbit $G$.x. A locally bounded $K$-category $R$ is called simply connected [29] if it is triangular (its quiver has no oriented cycles) and any Galois covering of $R$ is trivial. A locally bounded $K$-category $R$ is called standard if it admits a Galois covering $R^{\prime} \rightarrow R$ with $R^{\prime}$ simply connected. To every algebra $A$ we can attach the locally bounded $K$-category $R_{A}$ whose objects are formed by a complete set $E$ of pairwise orthogonal primitive idempotents of $A, R(e, f)=f A e, e, f \in E$, and the composition is induced by the multiplication in $A$. An algebra $A$ is called standard if the attached locally bounded $K$-category $R_{A}$ is standard.

1.3. For an algebra $A$ we shall denote by $\Gamma_{A}$ its Auslander-Reiten quiver [5], and by $\tau, \tau^{-}$the Auslander-Reiten translations $D \operatorname{Tr}$ and $\operatorname{Tr} D$, respectively [4]. We shall not distinguish between an indecomposable module, its isomorphism class and the vertex of $\Gamma_{A}$ corresponding to it. Moreover, we denote by $\Gamma_{A}^{s}$ the stable quiver of $\Gamma_{A}$ obtained from $\Gamma_{A}$ by removing the $\tau$-orbits of all indecomposable projective modules and the $\tau^{-}$-orbits of all indecomposable injective modules and the arrows attached to them.

1.4. A connected component $\mathscr{C}$ of the Auslander-Reiten quiver $\Gamma_{A}$ of $A$ is said to be sincere if for each indecomposable projective $A$-module $P$ there is an $A$-module $X$ whose isomorphism class is contained in $\mathscr{C}$ such that $\operatorname{Hom}_{A}(P, X) \neq 0$.

1.5. Recall from [30] that a component $\mathscr{C}$ of the Auslander-Reiten quiver $\Gamma_{A}$ of an algebra $A$ is called generalized standard if $\operatorname{rad}^{\dot{x}}(X, Y)=0$ for all modules $X$ and $Y$ from $\mathscr{C}$, where $\operatorname{rad}^{x}(\bmod (A))$ is the intersection of all powers of the Jacobson radical $\operatorname{rad}(\bmod (A))$ of $\bmod (A)$.

1.6. Following [13] a component $T$ of $\Gamma_{A}$ (respectively, of $\Gamma_{A}^{s}$ ) is said to be a tube if $T$ contains a cyclic path and its geometrical realization $|T|$ is homeomorphic to $S^{\prime} \times \mathbb{R}_{0}^{+}$ where $S^{1}$ is a unit circle and $\mathbb{R}_{1}^{+}$is the set of non-negative real numbers. For a tube $T$ the set $S^{1} \times\{0\}$ of $|T|$ called its mouth. A stable tube of rank $n \geq 1$ is a translation quiver of the form $\mathbb{Z} A_{\propto} /\left(\tau^{\prime \prime}\right)$. The stable tubes of rank one are said to be homogeneous. A family $\mathscr{T}=\left(T_{i}\right)_{i \in A}$ of tubes in $\Gamma_{A}$ (respectively, in $\Gamma_{A}^{s}$ ) is said to be standard if the full subcategory of $\bmod (A)(\operatorname{respectively,~} \bmod (A))$ formed by the objects of $\mathscr{T}$ is equivalent to the mesh-category $K(\mathscr{T})$ of $\mathscr{T}$ (see [27, Section 2]). 
1.7. Recall from [32] that a quasitube $T$ is a component in $\Gamma_{A}$ such that its stable part $T^{S}$ is a tube.

1.8. Let $\mathbf{n}=\left(n_{1}, n_{2}, \ldots, n_{t}\right)$ be a natural $t$-tuple. A family $\mathscr{T}=\left(T_{\lambda}\right)_{\lambda \in \mathbb{P}_{1} K}, \mathbb{P}_{1} K=K \cup$ $\{\infty\}$, of quasitubes in $\Gamma_{A}$ is said to be a tubular $\mathbb{P}_{1} K$-family of type $\mathbf{n}$ if the following condition is satisfied: the stable part $\mathscr{T}^{s}$ of $\mathscr{T}$ is a disjoint union of stable tubes $T_{\lambda}^{s}$, $\lambda \in \mathbb{P}_{1} K$, such that $t$ of these tubes have ranks $n_{1}, n_{2}, \ldots, n_{t}$ and the remaining ones are homogeneous.

1.9. Two components $\mathscr{C}_{1}, \mathscr{C}_{2}$ in $\Gamma_{A}$ are said to be orthogonal if for any $X \in \mathscr{C}_{1}$, $Y \in \mathscr{C}_{2}, \operatorname{Hom}_{A}(X, Y)=0=\operatorname{Hom}_{A}(Y, X)$.

1.10. A cycle in $\bmod (A)$ is a sequence $M_{0} \rightarrow M_{1} \rightarrow \cdots \rightarrow M_{n}=M_{0}$ of nonzero non-isomorphisms between indecomposable $A$-modules. Following [33] $A$ is said to be cycle-finite if for every cycle in $\bmod (A)$ all of morphisms on this cycle do not belong to $\operatorname{rad}^{x}(\bmod (A))$.

1.11. Following $[7,17]$ we shall say that a $\operatorname{module} Z$ in $\bmod (A)$ is a tilting (respectively, cotilting) module if it satisfies the following conditions:

(1) $\operatorname{Ext}_{A}^{2}(Z,-)=0$; (respectively, $\left.\operatorname{Ext}_{A}^{2}(-, Z)=0\right)$;

(2) $\operatorname{Ext}_{A}^{1}(Z, Z)=0$;

(3) the number of non-isomorphic indecomposable summands of $Z$ equals the rank of the Grothendieck group $K_{0}(A)$ of $A$.

Two algebras $A$ and $F$ are said to be tilting-cotilting equivalent if there exist a sequence of algebras $A=A_{0}, A_{1}, \ldots, A_{m}, A_{m+1}=F$ and a sequence of modules $Z_{A}^{i}$, $0 \leq i \leq m$, such that $A_{i+1}=\operatorname{End}_{A}\left(Z^{i}\right)$ and $Z^{i}$ is either a tilting or a cotilting module.

\section{Partially directed $K$-categories.}

2.1. Let $\Delta$ be a partially ordered set. A connected locally bounded $K$-category $R$ whose Auslander-Reiten quiver consists only of generalized standard components is defined to be $\Delta$-directed provided that there is a partition $\bigsqcup_{\delta \in \Delta} \mathscr{T}_{\delta}$ of the AuslanderReiten quiver $\Gamma_{R}$ onto the disjoint union of families $\mathscr{T}_{\delta}$ of pairwise orthogonal components such that the following conditions are satisfied:

(1) If $X \in \operatorname{add}\left(\mathscr{T}_{\delta_{1}}\right), Y \in \operatorname{add}\left(\mathscr{T}_{\delta_{2}}\right)$ and $\operatorname{Hom}_{R}(X, Y) \neq 0$ then $\delta_{1} \leq \delta_{2}$

(2) For every pair $\delta, \sigma \in \Delta$ such that $\delta<\sigma$ and every pair of components $\mathscr{C} \in \mathscr{T}_{\delta}$, $\mathscr{D} \in \mathscr{T}_{\sigma}$ there exists a finite sequence $\left\{\delta_{1}, \delta_{2}, \ldots, \delta_{r}, \delta_{r+1}=\sigma\right\} \subset \Delta$ such that $\delta \leq \delta_{1} \leq$ $\cdots \leq \delta_{r} \leq \delta_{r+1}$, and there exists a sequence of modules $\left\{X_{0}, X_{1}, \ldots, X_{r}, X_{r+1}\right\}$ such that $X_{0} \in \operatorname{add}(\mathscr{C}), X_{i} \in \operatorname{add}\left(\mathscr{T}_{i}\right), i=1, \ldots, r, X_{r+1} \in \operatorname{add}(\mathscr{D})$ and $\operatorname{Hom}_{R}\left(X_{j}, X_{j+1}\right) \neq 0$ for each $j=0,1, \ldots, r$.

(3) There are at most finitely many projective vertices in every $\mathscr{T}_{\delta}, \delta \in \Delta$.

A partition $\bigsqcup_{\delta \in \Delta} \mathscr{T}_{\delta}$ satisfying the above conditions is called $\Delta$-induced.

2.2. A $K$-automorphism $f: R \rightarrow R$ of a $\Delta$-directed locally bounded $K$-category $R$ is said to be $\Delta$-induced if there is an automorphism $f^{\prime}: \Delta \rightarrow \Delta$ of the partially ordered set $\Delta$ such that the induced by $f$ equivalence $F_{f}: \bmod (R) \rightarrow \bmod (R)$ satisfies the following condition: if $X \in \operatorname{add}\left(\mathscr{T}_{\delta}\right), \delta \in \Delta$, then $F_{f}(X) \in \operatorname{add}\left(\mathscr{T}_{f^{\prime}(\delta)}\right)$.

2.3. Lemma. Let $\Delta$ be a linearly ordered set. If $R$ is a $\Delta$-directed locally bounded $K$-category then every $K$-automorphism $f: R \rightarrow R$ is $\Delta$-induced.

Proof. Assume that $f: R \rightarrow R$ is a $K$-automorphism of a $\Delta$-directed locally bounded 
$K$-category $R$. Then the induced equivalence $F_{f}: \bmod (R) \rightarrow \bmod (R)$ preserves indecomposability of modules and irreducibility of morphisms. Thus $F_{f}$ maps connected components of $\Gamma_{R}$ onto connected components. First we shall show that $F_{f}$ yields a bijection between the set $\left\{\mathscr{T}_{\delta}\right\}_{\delta \in \Delta}$ of families of pairwise orthogonal components. In order to do this it is sufficient to show that if $\mathscr{C}, \mathscr{D} \in \mathscr{T}_{\delta}, \delta \in \Delta$, then $F_{f}(\mathscr{C}), F_{f}(\mathscr{D}) \in \mathscr{T}_{\delta^{\prime}}$. Suppose to the contrary that $F_{f}(\mathscr{C}) \in \mathscr{T}_{\delta^{\prime}}$ and $F_{f}(\mathscr{D}) \in \mathscr{T}_{\delta^{\prime \prime}}$ with $\delta^{\prime} \neq \delta^{\prime \prime}$. Without loss of generality we may assume that $\delta^{\prime}<\delta^{\prime \prime}$, for $\Delta$ is linearly ordered. Consider a sequence $X_{0}, X_{1}, \ldots, X_{r+1}$ of modules such that $X_{0} \in \operatorname{add}\left(F_{f}(\mathscr{C})\right), X_{i} \in \operatorname{add}\left(\mathscr{T}_{\delta_{i}}\right), i=1, \ldots, r, X_{r+1} \in \operatorname{add}\left(F_{f}(\mathscr{D})\right)$ with $\operatorname{Hom}_{R}\left(X_{i}, X_{j+1}\right) \neq 0, j=0,1, \ldots, r$ which exists by $2.1(2)$. Clearly $\delta^{\prime}<\delta_{1} \leq \ldots \leq \delta^{\prime \prime}$ by 2.1(1). Applying $F_{f^{-1}}$ we get that there is the sequence $F_{f^{-1}}\left(X_{0}\right), F_{f^{-1}}\left(X_{1}\right), \ldots, F_{f^{-1}}\left(X_{r}\right)$, $F_{f^{-1}}\left(X_{r+1}\right)$ of modules such that $\operatorname{Hom}_{R}\left(F_{f^{-1}}\left(X_{j}\right), F_{f^{-1}}\left(X_{j+1}\right)\right) \neq 0, j=0,1, \ldots, r$, and $F_{f^{-1}}\left(X_{0}\right) \in \mathscr{C}, F_{f^{-1}}\left(X_{r+1}\right) \in \mathscr{D}$. Then each indecomposable direct summand in $F_{f^{-1}}\left(X_{j}\right) \in$ $\mathscr{T}_{\delta_{j, l}}$ with $\delta_{j, l} \geq \delta$ by 2.1(1). Since $F_{f^{-1}}\left(X_{0}\right), F_{f^{-1}}\left(X_{r+1}\right) \in \mathscr{T}_{\delta}$ hence all $\delta_{j, l}=\delta$ which contradicts the orthogonality of all components in $\mathscr{T}_{\delta}$. Consequently, if $\mathscr{C}, \mathscr{D} \in \mathscr{T}_{\delta}$ then $F_{f}(\mathscr{C}), F_{f}(\mathscr{D}) \in \mathscr{T}_{\delta^{\prime}}$. Hence $F_{f}$ induces a bijection $f^{\prime}: \Delta \rightarrow \Delta$ given by the condition: $f^{\prime}(\delta)=\sigma$ iff there is an $R$-module $X \in \operatorname{add}\left(\mathscr{T}_{\delta}\right)$ such that $F_{f}(X) \in \operatorname{add}\left(\mathscr{T}_{\sigma}\right)$. In order to finish the proof we should show that $f^{\prime}: \Delta \rightarrow \Delta$ is an automorphism of the partially ordered set $\Delta$. Suppose that $\delta_{1} \leq \delta_{2}$ in $\Delta$. Then for some $X \in \operatorname{add}\left(\mathscr{T}_{\delta_{1}}\right)$ and some $Y \in \operatorname{add}\left(\mathscr{T}_{\delta_{2}}\right)$ we have $F_{f}(X) \in \operatorname{add}\left(\mathscr{T}_{f^{\prime}\left(\delta_{1}\right)}\right)$ and $F_{f}(Y) \in \operatorname{add}\left(\mathscr{T}_{f^{\prime}\left(\delta_{2}\right)}\right)$. But $R$ is $\Delta$-directed, hence there are: a sequence $\left\{\sigma_{1}, \ldots, \sigma_{r}, \sigma_{r+1}=\delta_{2}\right\} \subset \Delta$ with $\delta_{1} \leq \sigma_{1}$ and a sequence of $R$-modules $\left\{X_{0}, X_{1}, \ldots, X_{r}, X_{r+1}\right\}$ such that $X_{0} \in \operatorname{add}\left(\mathscr{T}_{\delta_{1}}\right), \quad X_{i} \in \operatorname{add}\left(\mathscr{T}_{\sigma_{i}}\right), i=1, \ldots, r, X_{r+1} \in$ $\operatorname{add}\left(\mathscr{T}_{\delta_{2}}\right)$ with $\operatorname{Hom}_{R}\left(X_{j}, X_{j+1}\right) \neq 0, j=0,1, \ldots, r$, by 2.1(2). Since $F_{f}$ is an equivalence we obtain by 2.1(1) the following sequence of inequalities: $f^{\prime}\left(\delta_{1}\right) \leq f^{\prime}\left(\sigma_{1}\right) \leq \ldots \leq f^{\prime}\left(\sigma_{r}\right) \leq$ $f^{\prime}\left(\delta_{2}\right)$. Consequently $f^{\prime}\left(\delta_{1}\right) \leq f^{\prime}\left(\delta_{2}\right)$ and $f^{\prime}$ is an automorphism of $\Delta$. Thus $f$ is $\Delta$-induced.

2.4. Following [32] a group $G$ of $K$-linear automorphisms of a locally bounded $K$-category is said to be admissible if its action on the objects is free and has finitely many orbits.

2.5. Corollary. Let $\Delta$ be a linearly ordered set. If $R$ is a $\Delta$-directed locally bounded $K$-category and $G$ is an admissible infinite cyclic group of $K$-linear automorphisms of $R$ then $G$ consists of $\Delta$-induced $K$-automorphisms.

Proof. Clear by Lemma 2.3 .

2.6. Lemma. Let $\Delta$ be an infinite linearly ordered set. If $R$ is a $\Delta$-directed locally bounded $K$-category with infinitely many objects and $G$ an admissible infinite cyclic group of $K$-linear automorphisms of $R$ then $G$ is a group of automorphisms of $\Delta$.

Proof. Under the assumptions of our lemma there is a map $(-)^{\prime}: \operatorname{Aut}_{K}(R) \rightarrow \operatorname{Aut}(\Delta)$ by Lemma 2.3, where $f^{\prime}: \Delta \rightarrow \Delta$ is an automorphism of $\Delta$ such that, for $f \in \operatorname{Aut}_{K}(R)$ and for every $X \in \operatorname{add}\left(\mathscr{T}_{\delta}\right), F_{f}(X) \in \operatorname{add}\left(\mathscr{T}_{f^{\prime}(\delta)}\right)$. Observe that the restriction $(-)_{G}^{\prime}$ of $(-)^{\prime}$ to a subgroup $G$ in Aut $_{K}(R)$ is a group homomorphism $(-)_{G}^{\prime}: G \rightarrow \operatorname{Aut}(\Delta)$. Indeed, $\left(\mathrm{id}_{R}\right)^{\prime}=\mathrm{id}_{\Delta}$ by the definition of $(-)^{\prime}$. If $g, f \in G$ then the induced equivalence $F_{s f}: \bmod (R) \rightarrow \bmod (R)$ is of the form $F_{g f}=F_{y} F_{f}$ and $(g f)^{\prime}=g^{\prime} f^{\prime}$. Finally $\left(f^{-1}\right)^{\prime}=\left(f^{\prime}\right)^{-1}$ is clear by the definition of $(-)^{\prime}$. Consequently $(-)_{G}^{\prime}: G \rightarrow \operatorname{Aut}(\Delta)$ is a group homomorphism for every subgroup $G$ 
of $\operatorname{Aut}_{K}(R)$. In order to finish the proof we should show that $(-)_{G}^{\prime}$ is a monomorphism when $G$ is an admissible infinite cyclic group. In this case, if $f^{\prime}=\mathrm{id}_{\Delta}$ and $f=g^{z}$ for some $z \in \mathbb{Z}$, where $g$ is a generator of $G$, then $\left(g^{\prime}\right)^{z}=\mathrm{id}_{\Delta}$ since $(-)_{G}^{\prime}$ is a homomorphism. But the induced (by $g^{z}$ ) equivalence $F_{g}=F_{g} \ldots F_{g}$ maps every family $\mathscr{T}_{\delta}, \delta \in \Delta$, onto itself. Thus $F_{g}$ : can map projective vertices from a family $\mathscr{T}_{\delta}$, for a fixed $\delta \in \Delta$, only onto projective vertices in the same family. Since $R$ is $\Delta$-directed there are at most finitely many projective vertices in every family $\mathscr{T}_{\sigma}, \sigma \in \Delta$, by 2.1(3). If $z$ is the minimal natural number such that $\left(g^{\prime}\right)^{z}=\mathrm{id}_{\Delta}$ then $\left(g^{\prime}\right)^{z}=g^{\prime} \ldots g^{\prime}$ and $g^{\prime} \neq \mathrm{id}_{\Delta}$ or $z=1$. If $z \neq 1$ then $g^{\prime}\left(\delta_{0}\right)<\delta_{0}$ or $g^{\prime}\left(\delta_{0}\right)>\delta_{0}$ for some $\delta_{0} \in \Delta$, because $\Delta$ is linearly ordered. In the first case we obtain that $\left(g^{\prime}\right)^{z}\left(\delta_{0}\right)<\left(g^{\prime}\right)^{z-1}\left(\delta_{0}\right)<\ldots<g^{\prime}\left(\delta_{0}\right)<\delta_{0}$ which contradicts the fact that $\left(g^{\prime}\right)^{z}=\mathrm{id}_{\Delta}$. In the second case one gets a similar contradiction. Consequently $z=1$. Then $G$ is not admissible, because it has infinitely many orbits of the objects of $R$. This proves the lemma.

2.7. Now we assume that there is given an infinite linearly ordered set $\Delta$ and an infinite cyclic group $G$ acting on $\Delta$ nontrivially and nontransitively. Fix an element $\delta_{0} \in \Delta$ and consider the set $\Delta \backslash G . \delta_{0}$ with the induced order by that in $\Delta$. Thus we have:

2.8. Lemma. $\Delta \backslash G . \delta_{0}$ is a disjoint union $\bigsqcup_{z \in z} \Delta_{z}$ of linearly ordered sets $\Delta_{z}, z \in \mathbb{Z}$, such that the following conditions are satisfied:

(1) If $z_{1} \leq z_{2}$ and $\delta_{1} \in \Delta_{z_{1}}, \delta_{2} \in \Delta_{z_{2}}$ then $\delta_{1} \leq \delta_{2}$.

(2) For any two $z_{1}, z_{2} \in \mathbb{Z}$ there is an isomorphism $\Delta_{z_{1}} \cong \Delta_{z_{2}}$ of partially ordered sets.

(3) There is a linear order on the set of the G-orbits $\Delta^{\prime}=\left(\Delta \backslash G . \delta_{0}\right) / G$ of $\Delta \backslash G . \delta_{0}$ such that $\Delta^{\prime} \cong \Delta_{0}$.

Proof. Under the assumptions and the notations of 2.7 consider a generator $f$ of $G$. Then either $\delta_{0}<f\left(\delta_{0}\right)$ or $f\left(\delta_{0}\right)<\delta_{0}$ since $f \neq \mathrm{id}_{\Delta}$ and $\Delta$ is linearly ordered. We shall consider only the case $\delta_{0}<f\left(\delta_{0}\right)$, because the other one is similar. In the case we obtain inductively that $f^{z}\left(\delta_{0}\right)<f^{z+1}\left(\delta_{0}\right)$ for every integer $z$. We put $\Delta_{z}=\left\{\delta \in \Delta: f^{z}\left(\delta_{0}\right)<\delta<\right.$ $f^{z+1}\left(\delta_{0}\right)$ \}. Since $G$ acts on $\Delta$ nontrivially and nontransitively hence $\Delta_{z} \neq \varnothing$ for any integer $z$. Since $\Delta_{z} \subset \Delta, z \in \mathbb{Z}$, consider in $\Delta_{z}$ the order of $\Delta$. Thus $\Delta_{z}$ is linearly ordered set obviously and $\Delta \backslash G . \delta_{0}=\bigsqcup_{z \in \mathbb{Z}} \Delta_{z}$ which proves (1).

In order to prove (2) observe that for any two integers $z_{1}, z_{2}$ such that $z_{1} \leq z_{2}$ the restriction of $f^{z_{2}-z_{1}}$ to $\Delta_{z_{1}}$ yields an isomorphism of $\Delta_{z_{1}}$ and $\Delta_{z_{2}}$.

Now consider the set $\Delta^{\prime}$ of the $G$-orbits of $\Delta \backslash G . \delta_{0}$. Observe that there is a bijection $h: \Delta_{0} \rightarrow \Delta^{\prime}$ given by the formula $h(\delta)=G$. $\delta$. This bijection induces a linear order on $\Delta^{\prime}$ such that $\Delta^{\prime} \cong \Delta_{0}$ as partially ordered sets.

2.9. Let $R$ be a $\Delta$-directed locally bounded $K$-category, where $\Delta$ is a given partially ordered set. Let $\Gamma_{R}=\bigsqcup_{\delta \in \Delta} \mathscr{T}_{\delta}$ be a $\Delta$-induced partition of $\Gamma_{R}$. Then a family $\mathscr{T}_{\delta_{0}}$, for some $\delta_{0} \in \Delta$, is said to be separating [28] if for any $\delta_{1}, \delta_{2} \in \Delta$ such that $\delta_{1} \leq \delta_{0} \leq \delta_{2}$ and any nonzero morphism $f: X \rightarrow Y$ such that $X \in \operatorname{add}\left(\mathscr{T}_{\delta_{1}}\right), \quad Y \in \operatorname{add}\left(\mathscr{T}_{\delta_{2}}\right)$ there are $Z \in \operatorname{add}\left(\mathscr{T}_{\delta_{0}}\right), f_{1}: X \rightarrow Z, f_{2}: Z \rightarrow Y$ with $f=f_{2} f_{1}$. Similarly, a component $\mathscr{C}$ in $\mathscr{T}_{\delta_{0}}$ is called separating if the above $Z \in \operatorname{add}(\mathscr{C})$. Clearly, if all components of $\mathscr{T}_{\delta_{0}}$ are separating then $\mathscr{T}_{\delta_{0}}$ is separating. The converse implication is not true in general.

2.10. Lemma. Let $\Delta$ be an infinite linearly ordered set. Let $R$ be a $\Delta$-directed locally bounded $K$-category that has a separating family $\mathscr{T}_{\delta_{10}}$. If $G$ is an admissible infinite cyclic group of $K$-linear automorphisms of $R$ then for any $g \in G, \mathscr{T}_{g^{\prime}\left(\delta_{0}\right)}$ is a separating family. 
Proof. Under the assumptions and the notations of our lemma consider a $\Delta$-induced partition $\Gamma_{R}=\bigsqcup_{\delta \in \Delta} \mathscr{T}_{\delta}$ and an element $g \in G$. We deduce from Lemma 2.3 that $g$ is $\Delta$-induced. Then there is an automorphism $g^{\prime}: \Delta \rightarrow \Delta$ of the partially ordered set $\Delta$ such that the induced by $g$ equivalence $F_{g}: \bmod (R) \rightarrow \bmod (R)$ satisfies the following implication: if $X \in \operatorname{add}\left(\mathscr{T}_{\delta}\right)$ then $F_{g}(X) \in \operatorname{add}\left(\mathscr{T}_{g^{\prime}(\delta)}\right), \delta \in \Delta$. Now suppose that $X \in \operatorname{add}\left(\mathscr{T}_{\delta_{1}}\right)$, $Y \in \operatorname{add}\left(\mathscr{T}_{\delta_{2}}\right)$ and $\delta_{1} \leq g^{\prime}\left(\delta_{0}\right) \leq \delta_{2}$. Let $f: X \rightarrow Y$ be any nonzero morphism. Then $\left(g^{\prime}\right)^{-1}\left(\delta_{1}\right) \leq \delta_{0} \leq\left(g^{\prime}\right)^{-1}\left(\delta_{2}\right) \quad$ and $\quad F_{g^{-1}}(X) \in \operatorname{add}\left(\mathscr{T}_{\left(g^{\prime}\right)^{-1}\left(\delta_{1}\right)}\right), \quad F_{g^{-1}}(Y) \in \operatorname{add}\left(\mathscr{T}_{\left(g^{\prime}\right)^{-1}\left(\delta_{2}\right)}\right)$, $F_{g^{-1}}(f): F_{g^{-1}}(X) \rightarrow F_{g^{-1}}(Y)$. Since $\mathscr{T}_{\delta_{0}}$ is separating, there are $Z \in \operatorname{add}\left(\mathscr{T}_{\delta_{0}}\right), f_{1}: F_{g^{-1}}(X) \rightarrow$ $Z, f_{2}: Z \rightarrow F_{g^{-1}}(Y)$ such that $F_{g^{-1}}(f)=f_{2} f_{1}$ by 2.8 . Therefore $F_{g}(Z) \in \operatorname{add}\left(\mathscr{T}_{g^{\prime}\left(\delta_{11}\right)}\right)$ and $f=F_{g}\left(f_{2}\right) F_{g}\left(f_{1}\right)$. Consequently, $\mathscr{T}_{g^{\prime}\left(\delta_{1}\right)}$ is a separating family of components, and our proof is finished.

\section{Selfinjective standard algebras of polynomial growth.}

3.1. The repetitive category (see $[\mathbf{1 8}]$ ) of a locally bounded category $R$ is the selfinjective locally bounded category $\hat{R}$ whose objects are pairs $(n, x)=x_{n}, x \in R, n \in \mathbb{Z}$, and $\hat{R}\left(x_{n}, y_{n}\right)=\{n\} \times R(x, y), \hat{R}\left(x_{n+1}, y_{n}\right)=\{n\} \times D R(y, x)$, and $\hat{R}\left(x_{p}, y_{q}\right)=0$ if $p \neq q$, $q+1$, where $D V$ denotes the dual space $\operatorname{Hom}_{K}(V, K)$.

3.2. A Euclidean algebra is a representation-infinite tilted algebra of Euclidean type having a complete slice in the preprojective component [28]. We shall use also tubular algebras in the sense of Ringel. For the basic definitions and results concerning Euclidean and tubular algebras we refer the reader to [28]. Recall that the extension type $\mathbf{n}_{A}$ of a Euclidean algebra $A$ is one of the following $(p, q), 1 \leq p \leq q,(2,2, m), m \geq 2,(2,3,3)$, $(2,3,4)$ or $(2,3,5)$. The extension type $\mathbf{n}_{A}$ of a tubular algebra $A$ is one of the following $(3,3,3),(2,4,4),,(2,3,6)$ or $(2,2,2,2)$. We shall call them, briefly, types.

3.3. The following theorem is the main result of [32].

THEOREM. Let $B$ be a standard, selfinjective $K$-algebra. Then $B$ is representationinfinite of polynomial growth if and only if $B$ is isomorphic to an algebra $\hat{A} / G$, where $A$ is either a Euclidean or a tubular algebra and $G$ is an admissible infinite cyclic group of $K$-linear automorphisms of $\hat{A}$.

3.4. Lemma. Let $A$ be a Euclidean algebra. Then $\hat{A}$ is a $\mathbb{Z}$-directed locally bounded $K$-category such that the $\mathbb{Z}$-induced partition $\Gamma_{\hat{A}}=\bigsqcup_{z \in \mathbb{Z}} \mathscr{T}_{z}$ satisfies the following conditions:

(1) For any even $z, \mathscr{T}_{z}$ consists of one connected component which contains at least one projective vertex, and whose stable Auslander-Reiten quiver is isomorphic to $\mathbb{Z} Q_{A}$, where $Q_{A}$ is the ordinary quiver of $A$. Moreover, $\mathscr{T}_{z}$ contains only finitely many projective vertices.

(2) For any odd $z, \mathscr{T}_{z}$ is a tubular $\mathbb{P}_{1} K$-family of quasitubes, whose stable Auslander-Reiten quiver is a tubular $\mathbb{P}_{1} K$-family of type $\mathbf{n}_{A}$.

(3) Every family $\mathscr{T}_{z}$ is a separating family of components.

Proof. See [32, 2.1].

3.5. Lemma. Let $A$ be a tubular algebra. Then $\hat{A}$ is a $\mathbb{Q}$-directed locally bounded $K$-category such that the $\mathbb{Q}$-induced partition $\Gamma_{\hat{A}}=\bigsqcup_{q \in \mathbb{Q}} \mathscr{T}_{q}$ satisfies the following conditions:

(1) For every $q \in \mathbb{Q} \backslash \mathbb{Z}, \mathscr{T}_{q}$ is a tubular $\mathbb{P}_{1} K$-family of type $\mathbf{n}_{A}$ of stable tubes. 
(2) For any $q \in \mathbb{Z}, \mathscr{T}_{q}$ is a tubular $\mathbb{P}_{1} K$-family of quasitubes which contains at least one and at most finitely many projective vertices. Moreover, the stable Auslander-Reiten quiver of $\mathscr{T}_{q}$ is a tubular $\mathbb{P}_{1} K$-family of type $\mathbf{n}_{A}$.

(3) Every family $\mathscr{T}_{q}$ is a separating family of components. Moreover, for $q \in(\mathbb{Q} \backslash \mathbb{Z})$ every component in $\mathscr{T}_{q}$ is a separating component.

Proof. See $[32,3.1]$ or $[19$, Section 3] or else $[16]$.

3.6. Lemma. Let $A$ be a Euclidean algebra. If $P, P^{\prime}$ are indecomposable projective $\hat{A}$-modules and $P \in \mathscr{T}_{z}, P^{\prime} \in \mathscr{T}_{z^{\prime}}, \operatorname{Hom}_{\hat{A}}\left(P, P^{\prime}\right) \neq 0$, then $z \leq z^{\prime} \leq z+4$.

Proof. The lemma is a consequence of [1, Proposition 2.5].

3.7. Lemma. Let $A$ be a tubular algebra. If $P, P^{\prime}$ are indecomposable projective $\hat{A}$-modules and $P \in \mathscr{T}_{q}, P^{\prime} \in \mathscr{T}_{q^{\prime}}, \operatorname{Hom}_{\hat{A}}\left(P, P^{\prime}\right) \neq 0$ then $q \leq q^{\prime} \leq q+3$.

Proof. The lemma is a consequence of [1, Proposition 2.5].

\section{The structure of the Auslander-Reiten quivers of some standard algebras.}

4.1. Let $R$ be a locally bounded $K$-category and $G$ an admissible group of $K$-linear automorphisms of $R$. Then there is a covering functor $F: R \rightarrow R / G$ induced by the action of $G$ on $R$ [14] which attaches to every object of $R$ its $G$-orbit. Then $F_{\lambda}$ denotes the induced push-down functor $F_{\lambda}: \bmod (R) \rightarrow \bmod (R / G)[8,14]$. If $G$ is torsion-free then $F_{\lambda}$ preserves indecomposables, Auslander-Reiten sequences, and maps projective $R$ modules onto projective $R / G$-modules, injective $R$-modules onto injective $R / G$-modules.

4.2. A locally bounded $K$-category $R$ is said to be locally support-finite $[\mathbf{1 0 , 1 1}]$ if for every indecomposable projective $R$-module $P$, the set of isomorphism classes of indecomposable projective $R$-modules $P^{\prime}$ such that there exists an indecomposable finite-dimensional $R$-module $M$ with $\operatorname{Hom}_{R}(P, M) \neq 0 \neq \operatorname{Hom}_{R}\left(P^{\prime}, M\right)$ is finite. If $R$ is locally support-finite $K$-category then $F_{\lambda}$ is dense and induces a bijection between the set (ind $(R) / \cong) / G$ of the $G$-orbits of the isomorphism classes of finite-dimensional indecomposable $R$-modules and the set $\operatorname{ind}(R / G) / \cong$ of the isomorphism classes of indecomposable finite-dimensional $R / G$-modules $[\mathbf{1 0}]$.

4.3. Proposition. Let $\Delta$ be an infinite linearly ordered set. If $R$ is a $\Delta$-directed locally support-finite $K$-category which has a separating family $\mathscr{T}_{\delta_{10}}$ and $G$ is an admissible infinite cyclic group of $K$-linear automorphisms of $R$ then there are a family $\mathscr{T}$ of components in $\Gamma_{R / G}$, a linearly ordered set $\mathbb{M} \neq \varnothing$ and a partition $\Gamma_{R / G} \backslash \mathscr{T}=\bigsqcup_{\mu \in \mathbb{M}} \mathscr{T}_{\mu}$ onto a disjoint union of families of components such that the following conditions are satisfied:

(1) For every two different components $\mathscr{C}, \mathscr{D} \in \mathscr{T}_{\mu}, \mu \in \mathbb{M}$, if $X \in \mathscr{C}, Y \in \mathscr{D}$ and $f: X \rightarrow Y$ is a nonzero morphism then there are $Z \in \operatorname{add}(\mathscr{T}), f_{1}: X \rightarrow Z, f_{2}: Z \rightarrow Y$ such that $f=f_{2} f_{1}$.

(2) If $\mu_{1}<\mu_{2}$ in $\mathbb{M}, Y \in \operatorname{add}\left(\mathscr{T}_{\mu_{1}}\right), X \in \operatorname{add}\left(\mathscr{T}_{\mu_{2}}\right)$ and $f: X \rightarrow Y$ is a nonzero morphism then there are $Z \in \operatorname{add}(\mathscr{T}), f_{1}: X \rightarrow Z, f_{2}: Z \rightarrow Y$ such that $f=f_{2} f_{1}$.

Moreover, if the induced action of $G$ on $\Delta$ is nontransitive and there is a separating family $\mathscr{T}_{\delta_{i}^{\prime}}$ of components such that $\delta_{0}<\delta_{0}^{\prime}<g\left(\delta_{0}\right)$ for the generator $g$ of $G$ then 
(3) For every two different components $\mathscr{C}, \mathscr{D} \in \mathscr{T}$, if $X \in \mathscr{C}, Y \in \mathscr{D}$ and $f: X \rightarrow Y$ is a nonzero morphism then there are $Z \in \operatorname{add}\left(\Gamma_{R / G} \backslash \mathscr{T}\right), f_{1}: X \rightarrow Z, f_{2}: Z \rightarrow Y$ such that $f=f_{2} f_{1}$.

Proof. Let $R$ be a $\Delta$-directed locally support-finite $K$-category, where $\Delta$ is an infinite linearly ordered set. Assume that $G$ is an admissible infinite cyclic group of $K$-linear automorphisms of $R$. Let $\Gamma_{R}=\bigsqcup_{\delta \in \Delta} \mathscr{T}_{\delta}$ be a $\Delta$-induced partition of $\Gamma_{R}$, and $\mathscr{T}_{\delta_{0}}$ a separating family of components, $\delta_{0} \in \Delta$. Denote by $C$ the quotient category $R / G$. Then $F: R \rightarrow C$ denotes the covering functor induced by the action of $G$ on $R . F_{\lambda}: \bmod (R) \rightarrow$ $\bmod (C)$ is the induced push-down functor which is dense by 4.2 , because $R$ is locally support-finite. Put $\mathscr{T}=F_{\lambda}\left(T_{\delta_{0}}\right)$. We deduce from Lemma 2.8 that there is a linearly ordered set $\mathbb{M}=\Delta^{\prime}$ such that $\Gamma_{C} \backslash \mathscr{T}$ has a partition $\bigsqcup_{\mu \in \mathbb{M}} \mathscr{T}_{\mu}$ onto a disjoint union of families of components, where $\mathscr{T}_{\mu}=F_{\lambda}\left(\mathscr{T}_{\delta}\right), \delta \in \Delta$ is such an element that $G . \delta=\mu$. Thus for any two different components $\mathscr{C}, \mathscr{D}$ from $F_{\lambda}\left(\mathscr{T}_{\delta}\right)$ if $X \in \mathscr{C}, Y \in \mathscr{D}$ and $f: X \rightarrow Y$ is a nonzero morphism then there are components $\mathscr{C}^{\prime} \in \mathscr{T}_{\delta_{1}}$ and $\mathscr{D}^{\prime} \in \mathscr{T}_{\delta_{2}}$ with $\delta_{1}, \delta_{2} \in G . \delta$ and there are $R$-modules $X^{\prime} \in C^{\prime}, Y^{\prime} \in \mathscr{D}^{\prime}$ with a nonzero morphism $f^{\prime}: X^{\prime} \rightarrow Y^{\prime}$ such that $F_{\lambda}\left(X^{\prime}\right)=X, F_{\lambda}\left(Y^{\prime}\right)=Y$ and $F_{\lambda}\left(f^{\prime}\right)=f$, for $F_{\lambda}$ is dense. But $R$ is $\Delta$-directed and $\left\{\mathscr{T}_{\delta}\right\}_{\delta \in \Delta}$ are the families of the $\Delta$-induced partition of $\Gamma_{R}$ hence $\delta_{1}<\delta_{2}$ by 2.1. Since $\delta_{1}$, $\delta_{2} \in G . \delta$ hence there is $\delta_{0}^{\prime} \in G . \delta_{0}$ such that $\delta_{1}<\delta_{0}^{\prime}<\delta_{2}$. Since $\mathscr{T}_{\delta_{0}}$ is a separating family hence we deduce from Lemma 2.10 that $\mathscr{T}_{\delta_{i}^{\prime}}$ is a separating family. Then there are $Z^{\prime} \in \operatorname{add}\left(\mathscr{T}_{\delta_{i}^{\prime}}\right), f_{1}^{\prime}: X^{\prime} \rightarrow Z^{\prime}, f_{2}^{\prime}: Z^{\prime} \rightarrow Y^{\prime}$ such that $f^{\prime}=f_{2}^{\prime} f_{1}^{\prime}$ by 2.9 . Clearly $F_{\lambda}\left(\mathscr{T}_{\delta_{i}^{\prime}}\right)=\mathscr{T}$. Thus $F_{\lambda}\left(Z^{\prime}\right)=Z \in \operatorname{add}(\mathscr{T})$ and $f=f_{2} f_{1}$, where $F_{\lambda}\left(f_{1}^{\prime}\right)=f_{1}: X \rightarrow Z$ and $F_{\lambda}\left(f_{2}^{\prime}\right)=f_{2}: Z \rightarrow Y$. Consequently condition (1) is proved.

Now assume that $\mu_{1}<\mu_{2}$ in $\mathbb{M}, Y \in \operatorname{add}\left(\mathscr{T}_{\mu_{1}}\right), X \in \operatorname{add}\left(\mathscr{T}_{\mu_{2}}\right)$ and $f: X \rightarrow Y$ is a nonzero morphism. Then there are $\delta_{1}, \delta_{2} \in \Delta$ such that $G . \delta_{1}=\mu_{1}, G . \delta_{2}=\mu_{2}$. Moreover, there are $X^{\prime} \in \operatorname{add}\left(\mathscr{T}_{\delta_{2}}\right), Y^{\prime} \in \operatorname{add}\left(\mathscr{T}_{\delta_{1}}\right)$ and $f^{\prime}: X^{\prime} \rightarrow Y^{\prime}$ such that $F_{\lambda}\left(X^{\prime}\right)=X$, $F_{\lambda}\left(Y^{\prime}\right)=Y$ and $F_{\lambda}\left(f^{\prime}\right)=f$. Since $R$ is $\Delta$-directed and $\Delta$ is linearly ordered hence $\delta_{2}<\delta_{1}$, because $\mu_{1}<\mu_{2}$. If there is no $\delta_{0}^{\prime} \in G$. $\delta_{0}$ such that $\delta_{2}<\delta_{0}^{\prime}<\delta_{1}$ then $\mu_{2}<\mu_{1}$ by the definition of the order in $\Delta^{\prime}$ (see the proof of Lemma 2.8), which contradicts the assumption that $\mu_{1}<\mu_{2}$. Thus there is $\delta_{0}^{\prime} \in G$. $\delta_{0}$ such that $\delta_{2}<\delta_{0}^{\prime}<\delta_{1}$. Therefore there are $Z^{\prime} \in \operatorname{add}\left(\mathscr{T}_{\delta_{i}^{\prime}}\right), f_{1}^{\prime}: X^{\prime} \rightarrow Z^{\prime}, f_{2}^{\prime}: Z^{\prime} \rightarrow Y^{\prime}$ such that $f^{\prime}=f_{2}^{\prime} f_{1}^{\prime}$ for $\mathscr{T}_{\delta_{i}^{\prime}}$ is separating by Lemma 2.10. Then there are $Z=F_{\lambda}\left(Z^{\prime}\right) \in \mathscr{T}, F_{\lambda}\left(f_{1}^{\prime}\right)=f_{1}: X \rightarrow Z, F_{\lambda}\left(f_{2}^{\prime}\right)=f_{2}: Z \rightarrow Y$ such that $f=f_{2} f_{1}$ which proves condition (2).

The proof of (3) is similar to that of (1), since we can use conditions (1), (2) for the family $F_{\lambda}\left(\mathscr{T}_{\delta_{0}}\right)$. We leave the details to the reader.

4.4. If $G$ is an admissible infinite cyclic group of $K$-linear automorphisms of a locally support-finite $K$-category $R$ then the quotient category $R / G$ is a finite-dimensional $K$-algebra. For a finite-dimensional $K$-algebra $H$ a family $\mathscr{T}$ of components in the Auslander-Reiten quiver $\Gamma_{H}$ is defined to be weakly separating if there is a non-empty linearly ordered set $\mathbb{M}$ and a partition $\Gamma_{H} \backslash \mathscr{T}=\bigsqcup_{\mu \in \mathbb{M}} \mathscr{T}_{\mu}$ of $\Gamma_{H} \backslash \mathscr{T}$ onto a disjoint union of families $\mathscr{T}_{\mu}$ of components such that the conditions (1)-(3) of Proposition 4.3 are satisfied. The set $\mathbb{M}$ will be called $\mathscr{T}$-induced.

4.5. THEOREM. If $B$ is a standard selfinjective representation-infinite $K$-algebra of polynomial growth then there is a weakly separating family $\mathscr{T}$ of components in $\Gamma_{B}$ with a $\mathscr{T}$-induced set $\mathbb{M}$ of one of the following forms:

$\Delta_{2 i+1}=\{1,2, \ldots, 2 i+1\}, i=0,1,2, \ldots$, with the order as in $\mathbb{N}$, 
$\Delta_{(0, i)}=(0, i) \cap \mathbb{Q}, i=1,2, \ldots$, with the order as in $\mathbb{Q}$.

Moreover, every family $\mathscr{T}_{\mu}$ of the partition $\Gamma_{B} \backslash \mathscr{T}=\bigsqcup_{\mu \in \mathbb{M}} \mathscr{T}_{\mu}$ is also a weakly separating family of components in $\Gamma_{B}$ with a $\mathscr{T}_{\mu}$-induced set which is isomorphic to $\mathbb{M}$.

Proof. Let $B$ be a standard selfinjective representation-infinite $K$-algebra of polynomial growth. Then $B \cong \hat{A} / G$, where $A$ is either a Euclidean or a tubular $K$-algebra and $G$ is an admissible infinite cyclic group of $K$-linear automorphisms of $\hat{A}$ by Theorem 3.3. If $A$ is a Euclidean $K$-algebra then $\hat{A}$ is a $\mathbb{Z}$-directed locally support-finite $K$-category which has a separating family $\mathscr{T}_{z}, z \in \mathbb{Z}$, by Lemma 3.4 . Thus $B$ has a weakly separating family $\mathscr{T}$ of components in $\Gamma_{B}$ by Proposition 4.3. Moreover, the $\mathscr{T}$-induced set $\mathbb{M}$ is of the form $\Delta_{2 i+1}=\{1,2, \ldots, 2 i+1\} . i=0,1,2, \ldots$ by its construction in the proof of Proposition 4.3 and by Lemma $3.4,2.8,2.10$. If $A$ is a tubular $K$-algebra then $\hat{A}$ is a $\mathbb{Q}$-directed locally support-finite $K$-category which has a separating family $\mathscr{T}_{q}, q \in \mathbb{Q}$, by Lemma 3.5 . Therefore $B$ has a weakly separating family $\mathscr{T}$ of components in $\Gamma_{B}$ by Proposition 4.3 . Moreover, the $\mathscr{T}$-induced set $\mathbb{M}$ is of the form $\Delta_{(0, i)}=(0, i) \cap \mathbb{Q}, i=1,2,3, \ldots$, by its construction in the proof of Proposition 4.3 and by Lemma 3.5, 2.8, 2.10. The last statement of the theorem is clear.

\section{Selfinjective algebras with Auslander-Reiten quivers all of whose components are generalized standard.}

5.1. Throughout this section we shall assume that $B$ is a standard selfinjective representation-infinite $K$-algebra of polynomial growth. Sometimes we shall use in the notations of Theorem 4.5 the convention that the family $\mathscr{T}$ has the index either $2 i+2$ if $M=\Delta_{2 i+1}$ or $i$ if $M=\Delta_{(0, i)}$.

5.2. Let ind $(B)$ denotes the full subcategory in $\bmod (B)$ formed by the indecomposable $B$-modules. For a nonzero non-isomorphism $f: X \rightarrow Y$ we define its $M$-length $l_{\mathrm{M}}(f)$ as follows: if $f=F_{\lambda}(\hat{f}): F_{\lambda}(\hat{X}) \rightarrow F_{\lambda}(\hat{Y})$, where $F_{\lambda}: \bmod (\hat{A}) \rightarrow \bmod (B)$ is the pushdown functor, then $\hat{X} \in \mathscr{T}_{\delta_{1}}, \hat{Y} \in \mathscr{T}_{\delta_{2}}$ and we put $l_{\mathbb{M}}(f)=\delta_{2}-\delta_{1}$. Furthermore, for a nonzero non-isomorphism $h: X \rightarrow Y$ in $\bmod (B)$ we have a decomposition $h: X_{1} \oplus \ldots \oplus$ $X_{n} \rightarrow Y_{1} \oplus \ldots \oplus Y_{m,}$, where $X_{l}, l=1, \ldots, n, Y_{j}, j=1, \ldots, m$, are indecomposable and $h=\left(h_{l j}\right)$ with $h_{l j}: X_{l} \rightarrow Y_{j}$. Then we define the M-length of $h$ as $l_{\mathbb{M}}(h)=\max _{l, j}\left\{l_{\mathbb{M}}\left(h_{l j}\right)\right\}$. Moreover for any isomorphism $f$ we put $l_{\mathbb{N}}(f)=0$. It is easy to verify that the above definition does not depend on the choice of pullings-up $\hat{X}, \hat{Y}$, and so M-length of a morphism is well-defined. For the algebra $B$ we define its $\mathbb{M}$-spread $\operatorname{spr}_{\mathbb{M}}(B)$ as $\sup _{0 \neq f}\left\{l_{M}(f)\right\}$.

5.3. Lemma. If $0 \neq f: X \rightarrow Y$ is a morphism in $\bmod (B)$ then there is a morphism $h: P \rightarrow P^{\prime}$ between indecomposable projective $B$-modules $P, P^{\prime}$ such that $l_{\mathrm{M}}(f) \leq l_{\mathrm{M}}(h)$.

Proof. In order to prove the lemma consider the composed morphism $h_{1}=w_{Y} f p_{X}$, where $p_{X}: P(X) \rightarrow X$ is a projective covering morphism and $w_{Y}: Y \rightarrow I(Y)$ is an embedding of $Y$ into its injective hull. Then $l_{\mathbb{M}}\left(h_{1}\right) \geq l_{\mathbb{M}}(f)$. If we decompose $h_{1}: P(X) \rightarrow I(Y)$ then there are an indecomposable direct summand $P$ in $P(X)$, an indecomposable direct summand $P^{\prime}$ in $I(Y)$ and a nonzero morphism $h: P \rightarrow P^{\prime}$ such that $l_{\mathbb{M}}(h)=l_{\mathbb{M}}\left(h_{1}\right) \geq l_{M}(f)$ by 5.2 .

5.4. If $G$ is an admissible infinite cyclic group of $K$-linear automorphisms of $\hat{A}$ and $g$ is a generator of $G$ then $g$ is a $\Delta$-induced automorphism by Corollary 2.5, where $\Delta=\mathbb{Q}$ or 
$\Delta=\mathbb{Z}$. Thus there is an automorphism $g^{\prime}: \Delta \rightarrow \Delta$ associated to $g$ as in 2.2 . Then a $\Delta$-stroke of $g$ is defined to be the natural number $\operatorname{st}_{\Delta}(g)=\left|g^{\prime}(\delta)-\delta\right|>0$ for any $\delta \in \Delta$. Clearly $\mathrm{st}_{\Delta}(\mathrm{g})$ is independent of the choice of $\delta \in \Delta$.

5.5. Proposition. Let $A$ be either a Euclidean or a tubular algebra. Let $G$ be an admissible infinite cyclic group of K-linear automorphisms of $\hat{A}, g$ a generator of $G$ and $B \cong \hat{A} / G$. Then the following conditions are equivalent:

(1) All components in $\Gamma_{B}$ are generalized standard.

(2) One of the following conditions holds:

(2a) $\mathrm{st}_{\Delta}(g)>\operatorname{spr}_{\mathbb{M}}(B)$.

(2b) $\operatorname{st}_{\Delta}(g)=\operatorname{spr}_{\mathbb{M}}(B)$ and for every indecomposable projective $B$-Module $P$ it holds that $P$ and the injective hull $I(\operatorname{top}(P))$ of top $(P)$ do not belong to to e same component in $\Gamma_{B}$.

Proof. First we shall show that condition (2) implies condition (1). Assume that $\operatorname{st}_{\Delta}(g)>\operatorname{spr}_{\mathbb{M}}(B)$. Suppose to the contr...y that there is a component $\mathscr{C}$ in $\Gamma_{B}$ which is not generalized standard. Then there a. $X, Y \in \mathscr{C}$ and $0 \neq f: X \rightarrow Y$ such that $f \in$ $\operatorname{rad}^{\infty}(\bmod (B))$. Since. $\hat{A}$ is locally suppe - finite, there are $\hat{X}, \hat{Y} \in \operatorname{ind}(\hat{A}), 0 \neq \hat{f}: \hat{X} \rightarrow \hat{Y}$ with $\hat{X} \in \mathscr{T}_{\delta_{1}}, \hat{Y} \in \mathscr{T}_{\delta_{2}}$ such that $F_{\lambda}(\hat{f})==f, F_{\lambda}(\hat{X})=X, F_{\lambda}(\hat{Y})=Y$. But all components in $\Gamma_{\hat{A}}$ are generalized standard hence $\delta_{1} \neq \delta_{2}$. We deduce from Corollary 2.5 that $g$ is $\Delta$-induced, so there is an automorphism $g^{\prime}: \Delta \rightarrow \Delta$ such that if $Z \in \mathscr{T}_{\delta}$ then $F_{g}(Z) \in \mathscr{T}_{g^{\prime}(\delta)}$, where $F_{g}$ is an automorphism of $\bmod (\hat{A})$ induced by $g$. Since $X, Y \in \mathscr{C}$ hence there is a natural number $n$ such that either $\left(g^{\prime}\right)^{n}\left(\delta_{1}\right)=\delta_{2}$ or $\left(g^{\prime}\right)^{-\prime \prime}\left(\delta_{1}\right)=\delta_{2}$. Then $\mathrm{st}_{\Delta}(g) \leq\left|\delta_{1}-\delta_{2}\right|$ and clearly $l_{\mathbb{M}}(f)=\left|\delta_{1}-\delta_{2}\right| \leq \operatorname{spr}_{\mathbb{M}}(B)$. Consequently $\operatorname{st}_{\Delta}(g) \leq \operatorname{spr}_{\mathbb{M}}(B)$ which contradicts our assumption. Thus every component in $\Gamma_{B}$ is generalized standard.

Now assume that $\mathrm{st}_{\Delta}(g)=\operatorname{spr}_{\mathbb{M}}(B)$ and for every indecomposable projective $B$ module $P$ it holds that $P$ and $I(\operatorname{top}(P))$ do not belong to the same component. Again suppose to the contrary that there is a component $\mathscr{C}$ in $\Gamma_{B}$ which is not generalized standard. Hence there are $X, Y \in \mathscr{C}$ and $0 \neq f: X \rightarrow Y$ such that $f \in \operatorname{rad}^{x}(\bmod (B))$. Then by Lemma 5.3 there are indecomposable projective $B$-modules $P, P^{\prime}$ and $0 \neq h: P \rightarrow P^{\prime}$ such that $h \in \operatorname{rad}^{x}(\bmod (B))$. Clearly we can choose $P$ as an indecomposable direct summand in $P(X)$ and $P^{\prime}$ as $I(\operatorname{top}(P))$. Then we can consider the following composed morphism $w \pi f p$, where $p: P \rightarrow X$ is induced by a covering morphism $P(X) \rightarrow X$, $\pi: Y \rightarrow Y / \operatorname{rad}(\operatorname{im}(f))$ is an epimorphism and $w: Y / \operatorname{rad}(\operatorname{im}(f)) \rightarrow P^{\prime}$ is induced by an embedding $Y / \operatorname{rad}(\operatorname{im}(f)) \rightarrow I(Y / \operatorname{rad}(\operatorname{im}(f)))$. Then $0 \neq w \pi f p \in \operatorname{rad}^{x}(\bmod (B))$. Since st $_{\lrcorner}(g)=\operatorname{spr}_{\mathbb{M}}(B)$ hence $P$ and $P^{\prime}$ belong to the same component which contradicts our assumption. Consequently, every component in $\Gamma_{B}$ is generalized standard.

Now assume that every component in $\Gamma_{B}$ is generalized standard. First we shall show that $\mathrm{st}_{\Delta}(g) \geq \operatorname{spr}_{\mathbb{M}}(B)$. Suppose to the contrary that $\mathrm{st}_{\Delta}(g)<\operatorname{spr}_{\mathbb{M}}(B)$. Then there is $0 \neq f: P \rightarrow P^{\prime}$ between indecomposable projective $B$-modules such that $l_{\mathbb{M}}(f)>\mathrm{st}_{\Delta}(g)$ by Lemma 5.3. Since $\hat{A}$ is locally support-finite hence there is $0 \neq \hat{f}: \hat{P} \rightarrow \hat{P}^{\prime}$ in $\bmod (\hat{A})$, where $\hat{P} \in \mathscr{T}_{\delta_{1}}, \hat{P}^{\prime} \in \mathscr{T}_{\delta_{2}}$ and $F_{\lambda}(\hat{P})=P, F_{\lambda}\left(\hat{P}^{\prime}\right)=P^{\prime}, F_{\lambda}(\hat{f})=f,\left|\delta_{1}-\delta_{2}\right|>\mathrm{st}_{\Delta}(g)$. But $\hat{A}$ is $\Delta$-directed, so $\delta_{2}>\delta_{1}$ and $\delta_{1}<g^{\prime}\left(\delta_{1}\right)<\delta_{2}$ or $\delta_{1}<\left(g^{\prime}\right)^{-1}\left(\delta_{1}\right)<\delta_{2}$, because $\Delta$ is linearly ordered. We shall consider only the first case since the other one is similar. If $A$ is tubular then there exists $\delta_{0} \in \mathbb{Q}$ such that $\delta_{1}<\delta_{0}<g^{\prime}\left(\delta_{1}\right)$ and $g^{\prime}\left(\delta_{1}\right)<g^{\prime}\left(\delta_{0}\right)<\delta_{2}$. Clearly $\hat{f}$ factorizes through a module $\hat{W}_{1}$ from $\operatorname{add}\left(\mathscr{T}_{\delta_{1}}\right)$ and through a module $\hat{W}_{2}$ from add $\left(\mathscr{T}_{g^{\prime}\left(\delta_{0}\right)}\right)$ by Lemma 3.5 . We can choose $\hat{W}_{1} \in \operatorname{add}(\mathscr{C})$ and $\hat{W}_{2} \in \operatorname{add}\left(F_{s}(\mathscr{C})\right)$, where $\mathscr{C}$ is a 
component from $\mathscr{T}_{\delta_{0}}$ by $\left[16\right.$, Section 2], because we can choose $\delta_{0} \in(\mathbb{Q} \backslash \mathbb{Z})$. Thus $\hat{f}=\hat{f}_{3} \hat{f}_{2} \hat{f}_{1}$, where $\hat{f}_{1}: \hat{P} \rightarrow \hat{W}_{1}, \hat{f}_{2}: \hat{W}_{1} \rightarrow \hat{W}_{2}, \hat{f}_{3}: \hat{W}_{2} \rightarrow \hat{P}^{\prime}$. Therefore $F_{\lambda}\left(\hat{f}_{2}\right): F_{\lambda}\left(\hat{W}_{1}\right) \rightarrow F_{\lambda}\left(\hat{W}_{2}\right)$ is a morphism from $\operatorname{rad}^{x}(\bmod (B))$ and $F_{\lambda}\left(\hat{W}_{1}\right), F_{\lambda}\left(\hat{W}_{2}\right) \in \operatorname{add}\left(F_{\lambda}(\mathscr{C})\right)$ which contradicts generalized standardness of the components in $\Gamma_{B}$. If $A$ is Euclidean then $\operatorname{st}_{\Delta}(g)>2$ by Lemma 3.6 and we obtain similarly that $F_{\lambda}\left(\mathscr{T}_{z}\right), z$ even, is not a generalized standard component in $\Gamma_{B}$ by Lemma 3.4. Consequently we have proved that $\mathrm{st}_{\Delta}(g) \geq \operatorname{spr}_{\mathbb{M}}(B)$. In order to finish our proof we should only show that if $\operatorname{st}_{\Delta}(g)=\operatorname{spr}_{\mathbb{M}}(B)$ then $P$ and $I($ top $(P))$ do not belong to the same component, where $P$ is any indecomposable projective $B$-module. Suppose to the contrary that there is an indecomposable projective $B$-module $P$ such that $P, I(\operatorname{top}(P)) \in \mathscr{C}$. Then the composed morphism $w p \neq 0$, where $p: P \rightarrow \operatorname{top}(P), w: \operatorname{top}(P) \rightarrow I(\operatorname{top}(P))$ and $w p \in \operatorname{rad}^{x}(\bmod (B))$. This contradicts the assumption that all components in $\Gamma_{B}$ are generalized standard. Thus the proposition follows.

5.6. Corollary. If $B$ is a selfinjective representation-infinite $K$-algebra with all components in $\Gamma_{B}$ generalized standard then the following conditions are satisfied:

(1) There is a partition $\Gamma_{B}=\bigsqcup_{v \in N} \mathscr{T}_{v}$ such that for every $v \in N, \mathscr{T}_{v}$ is a weakly separating family of components and $N \backslash\{v\}$ is isomorphic to $\Delta_{2 i+1}, i \geq 1$ or to $\Delta_{(0, i)}, i \geq 3$.

(2) $\operatorname{spr}_{N \backslash\{v\}}(B)=\left\{\begin{array}{lll}4 & \text { if } & N \backslash\{v\} \cong \Delta_{2 i+1} \\ 3 & \text { if } & N \backslash\{v\} \cong \Delta_{(0, i)}\end{array}\right.$

Proof. Since any selfinjective representation-infinite $K$-algebra $B$ with all components in $\Gamma_{B}$ generalized standard is a standard algebra of polynomial growth by [34] hence the corollary is clear by Theorem 4.5, Proposition 5.5 and Lemma 3.6, 3.7, 5.3.

5.7. Under the assumptions and the notations of 5.6 we have the following.

Corollary. If $0 \neq f: X \rightarrow Y$ is a morphism such that its coset modulo $\mathscr{P}(X, Y) \underline{f} \neq 0$ then

$$
l_{N \backslash\{v\}}(f) \leq\left\{\begin{array}{lll}
2 & \text { if } & N \backslash\{v\}=\Delta_{2 i+1}, i \geq 1, \\
1 \frac{1}{2} & \text { if } & N \backslash\{v\}=\Delta_{(0, i)}, i \geq 1 .
\end{array}\right.
$$

Proof. Observe that by [21] we have the following fact. For every nonprojective indecomposable $B$-module $X$ there is $0 \neq p: \tau^{-} \Omega X \rightarrow X$ such that for any $0 \neq \underline{f}: Y \rightarrow X$ there is $\underline{h}: \tau^{-} \Omega X \rightarrow Y$ with $\underline{p}=\underline{f} \underline{h}$, where $\Omega$ is the Heller's loop-space functor. Thus $l_{n \backslash v\}}(f)$ is maximal, where $f^{-} \neq 0$, iff $f$ acts from $\tau^{-} \Omega X$ into $X$. Then for any hereditary algebra $H$ a simple verification shows that $l_{N \backslash\{v\}}(f) \leq 2$. For canonical tubular algebra $E$ it follows from [23, Lemma 1.6] that $l_{N \backslash\{v\}}(f) \leq 1 \frac{1}{2}$. Since for a Euclidean algebra $A$ we have $\underline{\bmod }(\hat{A})$ is equivalent to $\underline{\bmod }(\hat{H})$ and for a tubular algebra $A$ we have $\bmod (\hat{A})$ is equivalent to $\underline{\bmod }(\hat{E})$, the corollary follows.

\section{Components of algebras stably equivalent to algebras whose components are generalized standard.}

6.1. Throughout this section we assume that $B$ is a selfinjective representationinfinite $K$-algebra such that all components in $\Gamma_{B}$ are generalized standard. Moreover, we 
assume that $C$ is an algebra which is stably equivalent to $B$ and $\Phi: \underline{\bmod }(B) \rightarrow \underline{\bmod }(C)$ is a fixed equivalence. We shall denote by $\Phi^{-1}$ a quasi-inverse of $\Phi$. It is well-known and easy to prove that $C$ is also selfinjective.

6.2. Lemma. Let $X, Y$ be two nonprojective indecomposable $C$-modules which belong to a component $\mathscr{C}$ in $\Gamma_{C}$. If $f \in \operatorname{rad}^{x}(X, Y)$ is a nonzero morphism then $\underline{f}=0$.

Proof. Under the assumptions and the notations of the lemma suppose to the contrary that there is $f \in \operatorname{rad}^{x}(\bmod (C))$ with $f \neq 0$. Then there is $0 \neq \underline{d}: X_{1} \rightarrow Y_{1}$ such that $\Phi\left(X_{1}\right)=X, \Phi\left(Y_{1}\right)=Y$ and $\Phi(\underline{d})=f$. Since the stable Auslander-Reiten quiver is invariant under taking stable equivalences by [6] hence there is a component $\mathscr{C}_{1}$ in $\Gamma_{B}$ such that $X_{1}$, $Y_{1} \in \mathscr{C}_{1}$. Observe that there is such $d^{\prime}: X_{1} \rightarrow Y_{1}$ that $\Phi\left(\underline{d}^{\prime}\right)=\underline{f}$ and $d^{\prime} \in \operatorname{rad}^{\infty}(\bmod (B))$. Indeed, if $f \in \operatorname{rad}^{x}(\bmod (C))$ then $f=f_{s}^{\prime} h_{-s} h_{-s+1} \ldots h_{-1} h_{0} h_{1} \ldots h_{r-1} h_{r} f_{r}^{\prime \prime}$, where $h_{j} \in$ $\operatorname{rad}(\bmod (C))$, so it is neither a split epimorphism nor a split monomorphism, $j=-s$, $-s+1, \ldots,-1,0,1, \ldots, r$. Moreover, for arbitrary large natural $s$ or $r, f$ has a decomposition of the above form. Thus we can choose $d^{\prime}=d_{s}^{\prime} t_{-s} t_{-s+1} \ldots t_{-1} t_{0} t_{1}$ $\ldots t_{r-1} t_{r} d_{r}^{\prime \prime}$ such that $\Phi\left(\underline{d s}_{s}^{\prime}\right)=\underline{f s}_{s}^{\prime}, \Phi\left(\underline{d_{r}^{\prime \prime}}\right)=\underline{f r}_{r}^{\prime \prime},\left(\Phi t_{j}\right)=\underline{h}_{\underline{j}}, j=-s, \ldots,-1,0,1, \ldots, r$. But passing from a decomposition $f_{s}^{\prime} h_{-s} \ldots h_{-1} h_{0} h_{1} \ldots h_{r} f_{r}^{\prime \prime}$ to $f_{s+1}^{\prime} h_{-s-1} h_{-s} \ldots h_{-1} h_{0} h_{1}$ $\ldots h_{r} h_{r+1} f_{r+1}^{\prime \prime}$ we decompose $f_{s}^{\prime}=f_{s+1}^{\prime} h_{-s+1}$ and $f_{r}^{\prime \prime}=h_{r+1} f_{r+1}^{\prime \prime}$ in the following way. If $f_{s}^{\prime}: \bigoplus_{i=1}^{n} Z_{i} \rightarrow Y$ with all $Z_{i}$ indecomposable, $i=1, \ldots, n$, then for every $i=1, \ldots, n$ there is an Auslander-Reiten sequence of the form $0 \rightarrow Z_{i} \stackrel{l_{i}}{\rightarrow} R_{i} \rightarrow \tau^{-1}\left(Z_{i}\right) \rightarrow 0$ by [4]. Clearly $f_{s}^{\prime}=\left(f_{s, 1}^{\prime}, \ldots, f_{s, n}^{\prime}\right)$, where $f_{s, i}^{\prime}: Z_{i} \rightarrow Y$. Then we know from [4] that there is a morphism $f_{s+1, i}^{\prime}: R_{i} \rightarrow Y$ such that $f_{s, i}^{\prime}=f_{s+1, i}^{\prime} l_{\text {. }}$. Thus putting

$$
f_{s+1}^{\prime}=\left(f_{s+1,1}^{\prime}, \ldots, f_{s+1, n}^{\prime}\right) \text { and } h_{-s+1}=\left(\begin{array}{ccccc}
l_{1} & 0 & 0 & \ldots & 0 \\
0 & l_{2} & 0 & \ldots & 0 \\
& . & . & . & . \\
0 & 0 & \ldots & 0 & l_{n}
\end{array}\right)
$$

we obtain a decomposition $f_{s}^{\prime}=f_{s+1}^{\prime} h_{-s-1}$ with $h_{-s-1} \in \operatorname{rad}(\bmod (C))$. Dually one decomposes $f_{r}^{\prime \prime}=h_{r+1} f_{r+1}^{\prime \prime}$. Thus for arbitrary large natural $s$ or $r, d^{\prime}$ has a decomposition of the above form. Therefore $d^{\prime} \in \operatorname{rad}^{x}(\bmod (B))$ and $\underline{d}^{\prime}=\underline{d}$ since $\Phi\left(\underline{d^{\prime}}\right)=\Phi(\underline{d})=f$. Since $\underline{\mathrm{d}^{\prime}} \neq 0$ hence $d^{\prime} \neq 0$, and so we get a contradiction to our assumption on generalized standardness of all components in $\Gamma_{B}$, which finishes the proof.

6.3. Lemma. If $0 \neq f: X \rightarrow Y$ is such a morphism that $f \in \operatorname{rad}^{\star}(\bmod (C))$ and $X$, $Y \in \operatorname{add}(\mathscr{C})$ are without projective direct summands, where $\mathscr{C}$ is a component in $\Gamma_{C}$, then $f$ factorizes through a module $W \in \operatorname{add}\left(\Gamma_{C} \backslash \mathscr{C}\right)$.

Proof. Assume that $0 \neq f: X \rightarrow Y$ with $X, Y \in \operatorname{add}(\mathscr{C})$ without projective direct summands, $f \in \operatorname{rad}^{x}(\bmod (C))$, where $\mathscr{C}$ is a component in $\Gamma_{C}$. Since $f \in \operatorname{rad}^{x}(\bmod (C))$ there is a decomposition $f=f_{s}^{\prime} h_{-s} \ldots h_{-1} h_{0} h_{1} \ldots h_{r} f_{r}^{\prime \prime}$, where $h_{j} \in \operatorname{rad}(\bmod (C))$, $j=-s, \ldots,-1,0,1, \ldots, r$. Moreover, for arbitrary large $s$ or $r, f$ has a decomposition of this form.

Passing from $f=f_{s}^{\prime} h_{-s} \ldots h_{-1} h_{0} h_{1} \ldots h_{r} f_{r}^{\prime \prime}$ to $f=f_{s+1}^{\prime} h_{-s-1} \ldots h_{-1} h_{0} h_{1} \ldots h_{r} f_{r}^{\prime \prime}$ we decompose $f_{s}^{\prime}=f_{s+1}^{\prime} h_{-s-1}$ as in the proof of Lemma 6.2. Passing from $f=$ $f_{s}^{\prime} h_{-s} \ldots h_{-1} h_{0} h_{1} \ldots h_{r} f_{r}^{\prime \prime}$ to $f=f_{s}^{\prime} h_{-s} \ldots h_{-1} h_{0} h_{1} \ldots h_{r+1} f_{r+1}^{\prime \prime}$ we decompose $f_{r}^{\prime \prime}=$ $h_{r+1} f_{r+1}^{\prime \prime}$ as in the proof of Lemma 6.2. 
Let $h_{j}: W_{j} \rightarrow W_{j-1}, j=-s+1, \ldots,-1,0,1, \ldots, r$. Clearly we may assume that all $W_{j}$ are in add $(\mathscr{C})$. Indeed, if $W_{j_{0}} \in \operatorname{add}\left(\Gamma_{C} \backslash \mathscr{C}\right)$ then $f$ factorizes through $W_{j_{0}}$ and the required condition (in the lemma) holds. If $W_{j_{0}}$ has a decomposition $W_{j_{1}}^{\prime} \oplus W_{j_{0}}^{\prime \prime}$ with $W_{j_{0}}^{\prime} \in \operatorname{add}(\mathscr{C})$ and $W_{j_{11}}^{\prime \prime} \in \operatorname{add}\left(\Gamma_{C} \backslash \mathscr{C}\right)$ then we can consider the morphism $f_{s}^{\prime} h_{-s} \ldots h_{j_{1}-1} h_{j_{0}}^{\prime} h_{j_{0}+1}^{\prime} h_{j_{0}+2}$ $\ldots h_{r} f_{r}^{\prime \prime}, \quad$ where $\quad h_{j_{1}+1}^{\prime}: W_{j_{1}+1} \rightarrow W_{j_{11}}^{\prime}, \quad h_{j_{1}+1}=\left(h_{j_{0}+1}^{\prime}, \quad h_{j_{0}+1}^{\prime \prime}\right), \quad h_{j_{0}+1}^{\prime \prime}: W_{j_{11}+1} \rightarrow W_{j_{10}^{\prime \prime} ;}^{\prime \prime} \quad h_{j_{0}}=$ $\left(\begin{array}{l}h_{j_{11}}^{\prime} \\ h_{j_{11}^{\prime}}^{\prime \prime}\end{array}\right): W_{j_{1}}^{\prime} \oplus W_{j_{11}^{\prime \prime}}^{\prime \prime} \rightarrow W_{j_{11}-1}$. In the same way we can assume that $\operatorname{im}\left(f_{s}^{\prime} h_{-s} \ldots h_{j}\right) \in \operatorname{add}(\mathscr{C})$, $j=-s, \ldots, 0, \ldots, r$.

Now consider the case when $r$ can be arbitrarily large. Then there is $j_{0} \in \mathbb{N}$ such that all $h_{j}$ are epimorphisms for $j \geq j_{0}$ by the construction of decompositions of $f$ given

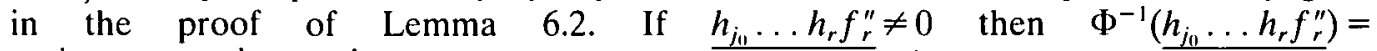
$\Phi^{-1}\left(h_{j_{0}}\right) \ldots \Phi^{-1}\left(\underline{h_{r}}\right) \Phi^{-1}\left(\underline{f_{r}^{\prime \prime}}\right) \neq 0$. Thus we can choose $h_{j}^{\prime}$ in $\operatorname{rad}(\bmod (\bar{B}))$ such that $h_{j}^{\prime}=\bar{\Phi}^{-1}\left(h_{j}\right), j=\bar{j}_{0}, \ldots, r$, because stable equivalences preserve irreducible morphisms. Hence there is a morphism $h$ in $\bmod (B)$ such that $\underline{h}=\Phi^{-1}\left(h_{j_{1}} \ldots h_{r} f_{r}^{\prime \prime}\right)$ and $h=$ $h_{j_{0}}^{\prime} \ldots h_{r}^{\prime} \bar{f}_{r}^{\prime \prime}$, where $\bar{f}_{r}^{\prime \prime}=\Phi^{-1}\left(f_{r}^{\prime \prime}\right)$. Consequently, $h \in \operatorname{rad}^{x}(\bmod (B))$ and $h: \Phi^{-1}(X) \rightarrow$ $\Phi^{-1}\left(W_{j_{0}}\right)$. This means that the component $\Phi^{-1}(\mathscr{C})$ in $\Gamma_{B}$ is not generalized standard which contradicts our assumption made in 6.1 .

If $h_{j_{1}} \ldots h_{r} f_{r}^{\prime \prime}=0$ then $h_{j_{0}} \ldots h_{r} f_{r}^{\prime \prime}$ factorizes through the projective cover $P$ of $W_{j_{0}}$. The same reasoning as in the first part of the proof shows that we may consider the largest direct summand $P^{\prime}$ in $P$ with $P^{\prime} \in \operatorname{add}(\mathscr{C})$. Then it is clear that the induced morphism $p: P^{\prime} \rightarrow W_{j_{0}}$ has a decomposition $p=h_{j_{0}} \ldots h_{r} p^{\prime \prime}$ for some $p^{\prime \prime}: P \rightarrow W_{r+1}$ by projectivity of $P$ since $h_{j}$ are epimorphisms for $j \geq j_{0}$. Thus $p \in \operatorname{rad}^{x}(\bmod (C))$. Furthermore, $p$ factorizes through $P^{\prime} / \operatorname{soc}\left(P^{\prime}\right)$, because $p=p_{1} \pi$ for an irreducible morphism $\pi: P^{\prime} \rightarrow P^{\prime} / \operatorname{soc}\left(P^{\prime}\right)$. It is obvious that $p_{1} \in \operatorname{rad}^{x}\left(\bmod (C)\right.$. Moreover $p_{1} \neq 0$, because the canonical epimorphism $q: P^{\prime} / \operatorname{soc}\left(P^{\prime}\right) \rightarrow \operatorname{top}\left(P^{\prime}\right)$ factorizes through $p_{1}$ hence $q=l p_{1}$ for some $l: W_{j_{0}} \rightarrow \operatorname{top}\left(P^{\prime}\right)$. But $q \neq 0$, because $q$ is an epimorphism. Hence $\underline{p}_{1} \neq 0$. Thus as in the case $h_{j_{n}} \ldots h_{r} f_{r}^{\prime \prime} \neq 0$ we get a contradiction to generalized standardness of the component $\Phi^{-1}(\mathscr{\mathscr { C }})$.

In the case when $s$ can be arbitrarily large dual arguments apply and the lemma follows.

6.4. Lemma. Let $\mathscr{C}$ be a component in $\Gamma_{C}$. If $X, Y \in \mathscr{C}$ and $0 \neq f: X \rightarrow Y$ such that $f \in \operatorname{rad}^{\infty}(\bmod (C))$ then there are $X_{1}, Y_{1} \in \mathscr{C}, W \in \operatorname{add}\left(\Gamma_{C} \backslash \mathscr{C}\right)$ and $h_{1}: X_{1} \rightarrow W, h_{2}: W \rightarrow Y_{1}$ such that $\underline{h_{1}} \neq 0$ and $\underline{h_{2}} \neq 0$.

Proof. Let $\mathscr{C}$ be a component in $\Gamma_{C}$. If $X, Y \in \mathscr{C}$ and $0 \neq f: X \rightarrow Y$ such that $f \in \operatorname{rad}^{x}(\bmod (C))$ then there is a module $W_{1} \in \operatorname{add}\left(\Gamma_{C} \backslash C\right)$ and there are $f_{1}: X \rightarrow W_{1}$, $f_{2}: W_{1} \rightarrow Y$ such that $f=f_{2} f_{1}$ by Lemma 6.3. If there is a direct summand $V$ in $\operatorname{im}(f)$ with $V \in \operatorname{add}\left(\Gamma_{C} \backslash \mathscr{C}\right)$ then the required condition holds for $W=V, X X_{1}=X, Y_{1}=Y, h_{1}: X \rightarrow V$ an epimorphism and $h_{2}: V \rightarrow Y$ a monomorphism. If $\operatorname{im}(f) \in \operatorname{add}(\mathscr{C})$ then consider $\operatorname{im}\left(f_{1}\right)$. If there is a direct summand $V_{1}$ in $\operatorname{im}\left(f_{1}\right)$ such that $V_{1} \in \operatorname{add}\left(\Gamma_{C} \backslash \mathscr{C}\right)$ then there is an epimorphism $t: \operatorname{im}\left(f_{1}\right) \rightarrow \operatorname{im}(f)$ and take $t_{1}: V_{1} \rightarrow Z_{1}$ as a restriction of $t$ to $V_{1}$ composed with a projection from $\operatorname{im}(f)$ onto an indecomposable direct summand in $\operatorname{im}(f)$ such that $p t_{1} \neq 0$, where $p: Z_{1} \rightarrow S$ is a projection and $S$ is a simple $C$-module. Thus the composed morphism $t=t_{1} t_{2}$ has the properties $t \neq 0, t \in \operatorname{rad}^{\infty}(\bmod (C))$, where $t_{2}: X \rightarrow V_{1}$ is an epimorphism. This contradicts Lemma 6.2. Hence $\operatorname{im}\left(f_{1}\right) \in \operatorname{add}(\mathscr{C})$. Applying the usual duality $D$ to $\operatorname{im}\left(f_{2}\right)$ we obtain similarly that $\operatorname{im}\left(f_{2}\right) \in \operatorname{add}(\mathscr{C})$. Since $f \neq 0$ there is an indecomposable direct summand $X_{1}$ in $\operatorname{im}\left(f_{1}\right)$ and there is an indecomposable direct 
summand $Y_{1}$ in $\operatorname{im}\left(f_{2}\right)$ such that $q v \neq 0$, where $v: X_{1} \rightarrow W_{1}$ is a monomorphism induced by $f_{1}$ and $q: W_{1} \rightarrow Y_{1}$ is an epimorphism induced by $f_{2}$. Consequently the required condition holds for $X_{1}, Y_{1}, W=W_{1}, h_{1}=v, h_{2}=q$ and the proof is finished.

6.5. Lemma. If $\mathscr{C}$ is a component in $\Gamma_{B}$ such that there are $X, Y \in \mathscr{C}$, an indecomposable $W \in \operatorname{add}\left(\Gamma_{B} \backslash \mathscr{C}\right)$ and $0 \neq \underline{h_{1}}: X \rightarrow W, 0 \neq \underline{h_{2}}: W \rightarrow Y$ then $\mathscr{C}$ does not contain a projective vertex.

Proof. Let $\mathscr{C}$ be a component in $\Gamma_{B}$ such that there are $X, Y \in \mathscr{C}$, an indecomposable $W \in \operatorname{add}\left(\Gamma_{B} \backslash \mathscr{C}\right)$ and $0 \neq \underline{h_{1}}: X \rightarrow W, 0 \neq \underline{h_{2}}: W \rightarrow Y$. Suppose to the contrary that $\mathscr{C}$ contains a projective vertex. We know from Corollary 5.6 that $\Gamma_{B}=\bigsqcup_{v \in N} \mathscr{T}_{v}$, where for every $v \in N, \mathscr{T}_{v}$ is a weakly separating family of components. Moreover, for every $v \in N$ we have that $N \backslash\{v\}$ is isomorphic to $\Delta_{2 i+1}, i \geq 1$, or $\Delta_{(0, i)}, i \geq 3$. Let $\mathscr{C} \in \mathscr{T}_{v_{i \mid}}, v_{0} \in N$. Let $W \in \mathscr{T}_{v_{1}}$. If $N \backslash\left\{v_{0}\right\} \cong \Delta_{(0, i)}, i \geq 3$, then $i=3$. Indeed, if $i>3$ then either $l_{N}\left(h_{1}\right)>1 \frac{1}{2}$ or $l_{N}\left(h_{2}\right)>1 \frac{1}{2}$ which contradicts Corollary 5.7. If $i=3$ then $l_{N}\left(h_{1}\right)=l_{N}\left(h_{2}\right)=1 \frac{1}{2}$, because $X, Y \in \mathscr{C}$ and $W \in \Gamma_{B} \backslash \mathscr{C}$. But we know from [6,21] and Corollary 5.7 that there is $0 \neq t: \tau^{-} \Omega W \rightarrow W$ such that $l_{N}(t)=1 \frac{1}{2}$. We infer by [6; Proposition 4.1] that the simple functor from $\bmod (B)$ to $\bmod (K)$ concentrated in $\tau^{-1} \Omega(W)$ is the socle of $\operatorname{Hom}_{B}(-, W)$. Thus there is $\underline{r}: \tau^{-1} \Omega(W) \rightarrow X$ such that $\underline{h_{1}} \underline{r}=\underline{t}$. Consequently, $\tau^{-1} \Omega(W) \in \mathscr{C}$. Dual arguments show that $\tau \Omega^{-1}(W) \in \mathscr{C}$.

For any indecomposable projective $B$-module $R$ we have the following AuslanderReiten sequence (see [5])

$$
0 \rightarrow \operatorname{rad}(R) \rightarrow R \oplus \operatorname{rad}(R) / \operatorname{soc}(R) \rightarrow R / \operatorname{soc}(R) \rightarrow 0
$$

because $B$ is selfinjective, and so every projective module is projective-injective and its top is simple as well as its socle. Thus $\tau \Omega^{-1}(\operatorname{soc}(R)) \cong \tau(R / \operatorname{soc}(R)) \cong \operatorname{rad}(R)$.

If there is a projective vertex $P$ in $\mathscr{C}$ then $\Omega^{-1}(\operatorname{rad}(P))$ belongs to the same component as $W$. But $\Omega^{-1}(\operatorname{rad}(P)) \cong \operatorname{top}(P)$. If we consider an injective envelope $I($ top $(P))$ of the simple $B$-module top $(P)$ then $I(\operatorname{top}(P))$ is indecomposable projectiveinjective and $\operatorname{soc}(I(\operatorname{top}(P))) \cong \operatorname{top}(P)$. Then $\Omega^{-1}(\operatorname{top}(P)) \cong I(\operatorname{top}(P)) / \operatorname{soc}(I(\operatorname{top}(P)))$ and $\tau \Omega^{-1}(\operatorname{top}(P)) \cong \operatorname{rad}(I(\operatorname{top}(P)))$ by the above Auslander-Reiten sequence. Since $\tau \Omega^{-1}(W) \in \mathscr{C}$ hence $\operatorname{rad}(I(\operatorname{top}(P))) \cong \tau \Omega^{-1}(\operatorname{top}(P)) \in \mathscr{C}$ which contradicts Proposition $5.5(2 \mathrm{~b})$ for all components in $\Gamma_{B}$ are generalized standard. The proof in the case when $N \backslash\left\{v_{0}\right\} \cong \Delta_{2 i+1}, i \geq 1$, is similar and we leave the details to the reader.

6.6. LemmA. If $\mathscr{C}$ is a component in $\Gamma_{C}$ which is not generalized standard, then $\mathscr{C}$ contains a projective vertex or a simple one.

Proof. Assume that $\mathscr{C}$ is a component in $\Gamma_{C}$ which is not generalized standard. Then there are $X, Y \in \mathscr{C}$ and $0 \neq f: X \rightarrow Y$ such that $f \in \operatorname{rad}^{x}(\bmod (C))$. Clearly we may assume that $X$ and $Y$ are not projective. Indeed, if $X$ is projective then $f=f_{2} f_{1}$, where $f_{1}: X \rightarrow X / \operatorname{soc}(X)$ is an epimorphism and $f_{2}: X / \operatorname{soc}(X) \rightarrow Y$. Moreover, we deduce from Lemma 6.3 that $f$ factorizes through a module $W \in \operatorname{add}\left(\Gamma_{C} \backslash \mathscr{C}\right)$. Thus $f_{2}$ factorizes through the same $W$, and so $f_{2} \in \operatorname{rad}^{x}(\bmod (C))$. Consequently we may consider $X / \operatorname{soc} X$ instead of $X$ and $f_{2}$ instead of $f$. Dual arguments show that $Y$ can be chosen nonprojective. We obtain from Lemma 6.2 that $f=0$. If $\operatorname{im}(f) \in \operatorname{add}\left(\Gamma_{C} \backslash \mathscr{C}\right)$ then $f=h_{2} h_{1}$, where $h_{1}: X \rightarrow$ $\operatorname{im}(f)$ is an epimorphism and $h_{2}: \operatorname{im}(f) \rightarrow Y$ is a monomorphism. Thus $h_{1}, h_{2} \neq 0$ by [27]. If $\Gamma_{B}=\cup_{v \in N} \mathscr{T}_{v}$ and $N \backslash\left\{v_{0}\right\}$ is isomorphic to $\Delta_{(o, i)}$, then $i=3$ and $l_{N}\left(\frac{2}{h_{1}}\right) \leq 1 \frac{1}{2}$, $l_{N}\left(h_{2}\right) \leq 1 \frac{1}{2}$ by Corollary 5.7. But we have an epimorphism $p: P(\operatorname{im}(f)) \rightarrow \operatorname{im}(f)$. 
Therefore there is a morphism $t: P(\operatorname{im}(f)) / \operatorname{soc}(P(\operatorname{im}(f))) \rightarrow X$ such that $t_{1}$ is an epimorphism. Thus $t h_{1} \neq 0$ and if $P(\operatorname{im}(f)) / \operatorname{soc}(P(\operatorname{im}(f)))$ does not belong to add $(\mathscr{C})$ then $l_{N}\left(t h_{1}\right)>1 \frac{1}{2}$ which contradicts Corollary 5.7. If $N \backslash\left\{v_{0}\right\}$ is isomorphic to $\Delta_{2 i+1}$, then $i=1$ and $l_{N}\left(h_{1}\right) \leq 2, l_{N}\left(h_{2}\right) \leq 2$. Repeating the above arguments we get that $P(\operatorname{im}(f)) \in \operatorname{add}(\mathscr{C})$.

If there is no direct summand $Z$ in $\operatorname{im}(f)$ such that $Z \in \operatorname{add}\left(\Gamma_{C} \backslash \mathscr{C}\right)$ then we infer by Lemma 6.4 that $f=h_{2} h_{1}$, where $h_{1}: X \rightarrow W, h_{2}: W \rightarrow Y$ and $W \in \operatorname{add}\left(\Gamma_{C} \backslash \mathscr{C}\right)$. Thus there is a submodule $W_{1}$ in $W$ such that there is an epimorphism $t_{1}: X \rightarrow W_{1}$ and there is an epimorphism $t_{2}: W_{1} \rightarrow \operatorname{im}(f)$. If $W_{1} \in \operatorname{add}(\mathscr{C})$ then consider the module $W_{2}=\operatorname{im}\left(h_{2}\right)$. There are monomorphisms $v_{1}: \operatorname{im}(f) \rightarrow W_{2}$ and $v_{2}: W_{2} \rightarrow Y$. Furthermore there is an epimorphism $q: W \rightarrow W_{2}$ such that $h_{2} h_{1}=v_{2} q h_{1}$. Since $v_{2} v_{1}$ is a monomorphism there is no nonzero direct summand $W_{2}^{\prime}$ in $W_{2}$ such that $W_{2}^{\prime} \in \operatorname{add}\left(\Gamma_{C} \backslash \mathscr{C}\right)$, because we get a contradiction to Lemma 6.2 otherwise. Consequently we can consider $q w$ instead of $f$, where $w: W_{1} \rightarrow W$ is a monomorphism. Thus, applying dual arguments to those in the case $\operatorname{im}(f) \in \operatorname{add}\left(\Gamma_{C}\right)$ $\mathscr{C})$, one obtains that $\mathscr{C}$ contains a simple module. In order to finish our proof we should consider the case when there is a direct summand $W_{1}^{\prime}$ in $W_{1}$ such that $W_{1}^{\prime} \in \operatorname{add}\left(\Gamma_{C} \backslash \mathscr{C}\right)$. But in that case we get a contradiction to Lemma 6.2 , because $t_{2} t_{1}$ is an epimorphism.

6.7. Proposition. Onè of the following conditions holds:

(1) All components in $\Gamma_{C}$ are generalized standard.

(2) There is a partition $\Gamma_{B}=\bigsqcup_{v \in N} \mathscr{T}_{v}$ such that for every $v_{0} \in N, N \backslash\left\{v_{0}\right\}$ is isomorphic to $\Delta_{(0,3)}$.

Proof. Fix a partition $\Gamma_{B}=\bigsqcup_{v \in N} \mathscr{T}_{v}$ described in Corollary 5.6. Assume for the proof of the proposition that $N \backslash\left\{v_{0}\right\}$ is isomorphic either to $\Delta_{(0, i)}, i>3$, or to $\Delta_{2 i+1}, i \geq 1$. Suppose to the contrary that there is a component $\mathscr{C}$ in $\Gamma_{C}$ which is not generalized standard. Then there are $X, Y \in \mathscr{C}, 0 \neq f: X \rightarrow Y$ such that $f \in \operatorname{rad}^{\infty}(\bmod (C))$. From Lemma 6.2 we have $f=0$. Furthermore we know from Lemma 6.3 that there is $W \in \operatorname{add}\left(\Gamma_{C} \backslash \mathscr{C}\right)$ and there are $f_{1}: X \rightarrow W, f_{2}: W \rightarrow Y$ such that $f=f_{2} f_{1}$. Using Lemma 6.4 we can choose $f_{1}, f_{2}, W$ in such a way that $f_{1} \neq 0 \neq f_{2}$. Therefore it is obvious by Corollary 5.7 that $\Gamma_{B}=\bigsqcup_{v_{\in} N} \mathscr{T}_{v}$ with $N \backslash\left\{v_{0}\right\}$ isomorphic to $\Delta_{3}$, because we assumed that it is not isomorphic to $\Delta_{(0,3)}$. But for $N \backslash\left\{v_{0}\right\}$ isomorphic to $\Delta_{3}$ we deduce from Lemma 3.4 that there is $v_{1} \in \Delta_{3}$ such that $\mathscr{T}_{v_{1}}$ consists of one component which contains projective vertices. Thus, if $P$ is a projective vertex in $\mathscr{T}_{v_{1}}$ and $I(\operatorname{top}(P))$ is the injective envelope of $\operatorname{top}(P)$ then $P, I(\operatorname{top}(P)) \in \mathscr{T}_{v_{1}}$ by 3.1 which contradicts (by Proposition 5.5) the assumption that all components in $\Gamma_{B}$ are generalized standard. Consequently the proposition is proved.

\section{Selfinjective algebras of type $\Delta_{(0,3)}$.}

7.1. Throughout this section we shall assume that $B$ is a representation-infinite selfinjective algebra such that all components in $\Gamma_{B}$ are generalized standard, and there is a partition $\Gamma_{B}=\bigsqcup_{v \in N} \mathscr{T}_{v}$, where $N \backslash\left\{v_{0}\right\}$ is isomorphic to $\Delta_{(0.3)}, v_{0} \in N$. Moreover $C$ is stably equivalent to $B$ and $\Phi: \underline{\bmod }(B) \rightarrow \underline{\bmod }(C)$ is a fixed equivalence.

\subsection{Lemma. $\Gamma_{C}$ contains a sincere tube which is generalized standard.}

Proof. We deduce from Lemma 6.6 that all components, which do not contain a projective module or a simple one, are generalized standard. If we carry over the partition $\Gamma_{B}=\bigsqcup_{v \in N} \mathscr{T}_{v}$ via $\Phi$ then we have $\Gamma_{C}=\bigsqcup_{v \in N} \mathscr{T}_{v}^{\prime}$. Without loss of generality we may 
assume that $N=[0,3) \cap \mathbb{Q}$. Since $C$ is a finite-dimensional algebra there are only finitely many rational numbers $q \in[0,3)$ such that $\mathscr{T}_{q}^{\prime}$ contains projective or simple modules. Thus we can choose a rational number $q_{0} \in[0,3)$ such that $q_{0} \notin\left\{0, \frac{1}{2}, 1,1 \frac{1}{2}, 2,2 \frac{1}{2}\right\}$ and all components in $\mathscr{T}_{q_{0}}^{\prime}$ are generalized standard. But we have that for every simple $C$-module $S$ either a projection $p: P(S) / \operatorname{soc}(P(S)) \rightarrow S$ or an injection $w: S \rightarrow \operatorname{rad}(I(S))$ factorizes through a module from add $\left(\mathscr{T}_{q_{1}}^{\prime}\right)$. But $\underline{p} \neq 0$ and $\underline{w} \neq 0$ hence for $\underline{\pi}=\Phi^{-1}(\underline{p}), \underline{u}=\Phi^{-1}(\underline{w})$ it holds that either $\pi$ or $u$ factorizes through a module from add( $\mathscr{C})$, where $\mathscr{C}$ ranges over all components in $\mathscr{T}_{q_{\mathrm{t}}}$ by Lemma 3.5(1). Therefore either $p$ or $w$ factorizes through a module from $\operatorname{add}\left(\mathscr{C}^{\prime}\right)$, where $\mathscr{C}^{\prime}$ ranges over all components in $\mathscr{T}_{\mathfrak{q}_{0}}^{\prime}$. Consequently every component in $\mathscr{T}_{q_{10}}^{\prime}$ is a generalized standard sincere tube and the lemma follows.

7.3. Fix a generalized standard sincere tube $T$ in $\Gamma_{C}$. Denotes by $\nabla$ the annihilator $\operatorname{ann}_{C}(T)$ of $T$ in $C$, which is the intersection of the annihilators of all modules from $T$. Clearly $\llbracket$ is nonzero, because $\operatorname{soc}(C) \subset \llbracket$. Moreover $\square \subset \operatorname{rad}(C)$ hence $T$ is a sincere tube in $\Gamma_{F}$, where $F=C / 0$.

7.4. Lemma. $F$ is cycle-finite.

Proof. Repeat the arguments from the proof of [23, Lemma 2.9].

7.5. Lemma. $C$ is a representation-infinite selfinjective standard algebra of polynomial growth.

Proof. It is clear that $C$ is representation-infinite selfinjective. The final part of the lemma can be deduced from a result of Skowroński and Yamagata [35] which says that if a selfinjective algebra $C$ has a generalized standard sincere stable tube $T$ such that $F=C / \operatorname{ann}_{C}(T)$ is cycle-finite then $C$ is standard of polynomial growth. Thus our lemma follows by Lemma 7.2 and Lemma 7.4.

\section{Proof of the main result.}

8.1. Let $B$ be a selfinjective representation-infinite algebra such that all components of $\Gamma_{B}$ are generalized standard. Let $C$ be an algebra which is stably equivalent to $B$ and let $\Phi: \underline{\bmod }(B) \rightarrow \underline{\bmod }(C)$ be a fixed equivalence.

8.2. Lemma. $C$ is a representation-infinite selfinjective standard algebra of polynomial growth.

Proof. We know from Proposition 6.7 and Lemma 7.5 that all components of $\Gamma_{C}$ are generalized standard or $C$ is a representation-infinite selfinjective standard algebra of polynomial growth. But in the case when all components of $\Gamma_{C}$ are generalized standard it follows from [34, Theorem 3.13] that $C$ is representation-infinite selfinjective standard algebra of polynomial growth.

8.3. Lemma. Let $\Delta$ be an infinite linearly ordered set and let $R$ be a $\Delta$-directed locally bounded $K$-category. Let $G$ be an admissible infinite cyclic group of $K$-linear automorphisms of $R$ such that for every nonprojective $X \in \mathscr{T}_{\delta_{11}}, \delta_{o} \in \Delta$, and every indecomposable $Y$ if $\underline{\operatorname{Hom}}_{R}(X, Y) \neq 0$ then $Y \in \mathscr{T}_{\delta_{1}}$ with $\delta_{0} \leq \delta_{1}<g^{\prime}\left(\delta_{0}\right)$, where $\Gamma_{R}=\bigsqcup_{\delta \in \Delta} \mathscr{T}_{\delta}$ is a $\Delta$-induced partition of $\Gamma_{R}$ and $g$ is a generator of $G$ which satisfies $\delta<g^{\prime}(\delta), \delta \in \Delta$. If $F_{\lambda}: \bmod (R) \rightarrow$ $\bmod (R / G)$ is an induced by the action of $G$ on $R$ push-down functor and $\underline{\operatorname{Hom}}_{R}(U, V) \neq$ $0, U, V \in \operatorname{ind}(\mathrm{R})$, then $\underline{\operatorname{Hom}}_{k}(U, V) \cong \underline{\operatorname{Hom}}_{R / C_{i}}\left(F_{\lambda}(U), F_{\lambda}(V)\right)$ as $K$-linear spaces. 
Proof. Under the assumptions and the notations of the lemma observe that if $f: U \rightarrow V$ is such a morphism in $\bmod (R)$ that $\underline{f} \neq 0$ then $F_{\lambda}(f): F_{\lambda}(U) \rightarrow F_{\lambda}(V)$ satisfies $F_{\lambda}(f) \neq 0$. Indeed, $F_{\lambda}$ preserves projective modules and factorizations of morphisms through projective modules. Let $U, V \in$ ind $(R)$ and $U \in \mathscr{T}_{\delta_{10}}, \delta_{0} \in \Delta$. If $\underline{\operatorname{Hom}}_{R}(U, V) \neq 0$ then $V \in \mathscr{T}_{\delta_{1}}$ with $\delta_{0} \leq \delta_{1}<g^{\prime}\left(\delta_{0}\right)$ by the assumption. But the $G$-orbit of $V$ intersects with $\sqcup_{\delta \in\left[\delta_{i, S^{\prime}}\left(\delta_{\delta}\right)\right]} \mathscr{T}_{\delta}$ in exactly one point $V$. Thus the lemma follows.

8.4. Let $\Delta$ be an infinite linearly ordered set. An admissible infinite cyclic group $G$ of $K$-linear automorphisms of a $\Delta$-directed locally bounded $K$-category $R$ is called stably admissible if it satisfies the following condition: for every $\Delta$-induced partition of $\Gamma_{R}$ and for every nonprojective $X \in \mathscr{T}_{\delta_{11}}$ and every nonprojective indecomposable $R$-module $Y$ if $\underline{\operatorname{Hom}}_{R}(X, Y) \neq 0$ then $Y \in \mathscr{T}_{\delta_{1}}$ with $\delta_{0} \leq \delta_{1}<g^{\prime}\left(\delta_{0}\right)$, where $g$ is a generator of $G$ such that $g^{\prime}(\delta)>\delta, \delta \in \Delta$.

8.5. Proposition. Let $\Delta$ be an infinite linearly ordered set. Let $R_{1}, R_{2}$ be two selfinjective $\Delta$-directed locally bounded $K$-categories. Let $G_{i}$ be a stably admissible group of $K$-linear automorphisms of $R_{i}, i=1,2$. If $R_{1} / G_{1}$ is stably equivalent to $R_{2} / G_{2}$ then $R_{1}$ is stably equivalent to $R_{2}$.

Proof. Under the assumptions and the notations of the proposition assume that (D: $\bmod \left(R_{1} / G_{1}\right) \rightarrow \bmod \left(R_{2} / G_{2}\right)$ is a fixed equivalence. Let $F_{i, \lambda}: \bmod \left(R_{i}\right) \rightarrow \bmod \left(R_{i} / G_{i}\right)$, $i=1,2$ be an induced by the action of $G_{i}$ on $R_{i}$ push-down functor. We shall construct an equivalence $\Psi: \bmod \left(R_{1}\right) \rightarrow \underline{\bmod }\left(R_{2}\right)$ as follows. Since $\Phi$ preserves the stable AuslanderReiten quiver $\Gamma_{R_{1} / G_{i}}^{s}$ and $F_{i, \lambda}$ maps components of $\Gamma_{R_{i}}$ onto components of $\Gamma_{R_{i} / G_{i},} i=1,2$, hence we can carry over the partition of $\Gamma_{R_{1}}$ onto the partition of $\Gamma_{R_{2}}$. Thus we may assume that if nonprojective $X, Y \in \mathscr{T}_{\delta_{1}} \subset \Gamma_{R_{1}}$ then there is $\delta_{2} \in \Delta$ such that there are nonprojective $X^{\prime}, Y^{\prime} \in \mathscr{T}_{\delta_{2}}^{\prime} \subset \Gamma_{R_{2}}$ such that $\Phi\left(F_{1, \lambda}(X)\right)=F_{2 . \lambda}\left(X^{\prime}\right)$ and $\Phi\left(F_{1, \lambda}(Y)\right)=$ $F_{2, \lambda}\left(Y^{\prime}\right)$ ). Fix a family $\mathscr{T}_{\delta_{0}}$ in $\Gamma_{R_{1}}, \delta_{0} \in \Delta$. Choose a family $\mathscr{T}_{\delta_{1}}^{\prime}$ in $\Gamma_{R_{2}}$ in such a way that for every nonprojective $X \in \mathscr{T}_{\delta_{01}}$ there is $Y \in \mathscr{T}_{\delta_{1}}^{\prime}$ satisfying $\Phi\left(F_{1, \lambda}(X)\right)=F_{2, \lambda}(Y)$. It is possible to do this by the above choice of the partition $\bigsqcup_{\delta_{\epsilon} \Delta} \mathscr{T}_{\delta}^{\prime}$ of $\Gamma_{R_{2}}$. Then for every $X \in \mathscr{T}_{\delta_{0}}$ we put $\Psi(X)=Y$ with $Y \in \mathscr{T}_{\delta_{1}}^{\prime}$ as above. If $g_{i}, i=1,2$ is a generator of $G_{i}$ satisfying the condition in 8.4 and $g_{i}^{\prime}: \Delta \rightarrow \Delta$ is an automorphism induced by $g_{i}, i=1,2$, then clearly $g_{1}^{\prime}=g_{2}^{\prime}$ by the choice of the partition of $\Gamma_{R_{2}}$. Therefore for any $z \in \mathbb{Z}$ if $X \in \mathscr{T}_{\left(g_{1}^{\prime}\right) ;\left(\delta_{11}\right)}$ then we can find the only $Y \in \mathscr{T}_{\left(g^{\prime}\right)^{*}\left(\delta_{2}\right)}^{\prime}$ such that $\Phi\left(F_{1 . \lambda}(X)\right)=F_{2, \lambda}(Y)$. We put $\Psi(X)=Y$ again. Moreover, for every $\delta \in\left[\delta_{0}, g_{1}^{\prime}\left(\delta_{0}\right)\right]$ there is exactly one $\sigma \in\left[\delta_{1}, g_{2}^{\prime}\left(\delta_{1}\right)\right]$ such that for every nonprojective $X \in \mathscr{T}_{\delta}$ there is exactly one $Y \in \mathscr{T}_{\sigma}^{\prime}$ with $\Phi\left(F_{1, \lambda}(X)\right)=F_{2, \lambda}(Y)$. Again for $X \in \mathscr{T}_{\delta}$ we put $\Psi(X)=Y$, where $Y \in \mathscr{T}_{\sigma}^{\prime}$ and $\Phi\left(F_{1, \lambda}(X)\right)=F_{2, \lambda}(Y)$. Now we can prolongate $\Psi$ for the objects of the shifted families $\mathscr{T}_{\left(g^{\prime}\right)^{z}(\delta)}, z \in \mathbb{Z}, \delta \in\left[\delta_{0}, g_{1}^{\prime}\left(\delta_{0}\right)\right]$. In this way we have defined $\Psi$ for the indecomposable objects. Furthermore we prolongate $\Psi$ for the objects of $\bmod \left(R_{1}\right)$ additively. Moreover, for every $0 \neq f: X \rightarrow Y$ in $\bmod \left(R_{1}\right)$ we define $\Psi(\underline{f})=\underline{h}: \Psi \overline{(X)} \rightarrow \Psi(Y)$, where $\left.\Phi F_{1, \lambda}(f)\right)=F_{1, \lambda}(h)$. It is easy to verify that $\Psi$ is a well-defined functor. Clearly $\Psi$ is dense by its definition. Finally $\Psi$ is fully faithful by Lemma 8.3 and the proposition follows.

Proof of Theorem. Let $B$ be a representation-infinite selfinjective algebra such that all components of $\Gamma_{B}$ are generalized standard. Then $B$ is standard of polynomial growth by [34, Theorem 3.13] and so $B \cong \hat{A} / G$ by Theorem 3.3, where $A$ is Euclidean or tubular 
and $G$ is an admissible infinite cyclic group of $K$-linear automorphisms of $\hat{A}$. If $C$ is stably equivalent to $B$ then $C$ is also a representation-infinite selfinjective standard algebra of polynomial growth by Lemma 8.2. Thus $C \cong \hat{A}_{1} / G_{1}$, where $A_{1}$ is Euclidean or tubular and $G_{1}$ is an admissible infinite cyclic group of $K$-linear automorphisms of $\hat{A}_{1}$. It is clear that $A$ is Euclidean (respectively, tubular) iff $A_{1}$ is Euclidean (respectively, tubular). It is obvious by Corollary 5.7 that $G, G_{1}$ are stably admissible. Then we deduce from Proposition 8.5 that $\hat{A}$ and $\hat{A}_{1}$ are stably equivalent. Thus $A, A_{1}$ are tilt-cotilting equivalent algebras by [3] and they have the same number of pairwise non-isomorphic simple modules by [17]. Furthermore, if $g$ is a generator of $G$ and $g_{1}$ is a generator of $G_{1}$ then $\mathrm{st}_{\Delta}(g)=\mathrm{st}_{\Delta}\left(g_{1}\right)$. Consequently, $B$ and $C$ have the same number of pairwise non-isomorphic simple modules which finishes the proof.

Acknowledgement. The author would like to thank the referee for all comments which were very helpful.

\section{REFERENCES}

1. I. Assem, J. Nehring and W. Schewe, Fundamental domains and duplicated algebras, Canadian Math. Soc. Conference Proceedings 11 (1991), 25-51.

2. I. Assem and A. Skowroński, On some class of simply connected algebras, Proc. London Math. Soc. 56 (1988), 417-450.

3. I. Assem and A. Skowroński, Algebras with cycle-finite derived categories, Math. Ann. 280 (1988), 441-463.

4. M. Auslander and I. Reiten, Representation theory of artin algebras III, Comm. Algebra 3 (1975), 239-294.

5. M. Auslander and I. Reiten, Representation theory of artin algebras IV, Comm. Algebra 5 (1977), 443-518.

6. M. Auslander and I. Reiten, Representation theory of artin algebras VI, Comm. Algebra 6 (1978), 257-300.

7. K. Bongartz, Tilted algebras in: Representations of Algebras, Lecture Notes in Math. 903 (Springer, Berlin, 1981), 26-38.

8. K. Bongartz and P. Gabriel, Covering spaces in representation theory, Invent. Math. 65 (1981/82), 331-378.

9. O. Bretscher, C. Läser and C. Riedtmann, Selfinjective and simply connected algebras, Manuscr. Math. 36 (1981/82), 253-307.

10. P. Dowbor and A. Skowroński, On Galois coverings of tame algebras, Arch. Math. 44 (1985), 522-529.

11. P. Dowbor and A. Skowroński, Galois coverings of representation-infinite algebras, Comment. Math. Helv. 62 (1987), 311-337.

12. Y. A. Drozd, Tame and wild matrix problems, in Representations and quadratic forms, (Kiev, Akad. Nauk USSR, 1979), 39-74.

13. G. d'Este and C. M. Ringel, Coherent tubes, J. Algebra 87 (1984), 150-201.

14. P. Gabriel, The universal cover of a representation-finite algebra, in Representations of Algebras, Lecture Notes in Math. 903 (Springer, Berlin, 1981), 68-105.

15. D. Happel, On the derived category of a finite-dimensional algebra, Comment. Math. Helv. 62 (1987), 339-389.

16. D. Happel and C. M. Ringel, The derived category of a tubular algebra, Lecture Notes in Math. 1177 (Springer, Berlin, 1986), 156-180.

17. D. Happel and C. M. Ringel, Tilted algebras, Trans. Amer. Math. Soc. 274 (1982), 339-443.

18. D. Hughes and J. Waschbüsch, Trivial extensions of tilted algebras, Proc. London Math. Soc. 46 (1983), 347-364.

19. J. Nehring and A. Skowroński, Polynomial growth trivial extensions of simply connected algebras, Fund. Math. 132 (1989), 117-134. 
20. L. Peng and J. Xiao, Invariability of trivial extensions of tilted algebras under stable equivalence, J. London Math. Soc. (2) 52 (1995), 61-72.

21. Z. Pogorzaly, On the stable Grothendieck groups, Canadian Mathematical Society, Conference Proceedings, 14 (1993), 393-406.

22. Z. Pogorzały, Algebras stably equivalent to the trivial extensions of hereditary and tubular algebras, preprint, Toruń (1994).

23. Z. Pogorzaly and A. Skorwoński, Symmetric algebras stably equivalent to the trivial extensions of tubular algebras, J. Algebra, 181 (1996), 95-111.

24. C. Riedtmann, Algebren, Darstellungköcher, Überlagerungen and zurück, Comment. Math. Helv. 55 (1980), 199-224.

25. C. Riedtmann, Representation-finite selfinjective algebras of type $\mathbb{A}_{n}$, Lecture Notes in Math. 832 (Springer, Berlin, 1980), 449-520.

26. C. Riedtmann, Representation-finite selfinjective algebras of class $\mathbb{D}_{n}$, Compos. Math. 49 (1983), 231-282.

27. C. M. Ringel, Representation theory of finite-dimensional algebras, in Representations of algebras, Proc. Durham Symposium 1985, London Math. Soc. Lecture Notes Series 116, (Cambridge U.P.) $7-79$.

28. C. M. Ringel, Tame algebras and integral quadratic forms, Lecture Notes in Math. 1099 (Springer, Berlin, 1984).

29. A. Skowroński, Algebras of polynomial growth, in Banach Center Publications, 26 (PWN, Warszawa, 1990), 535-568.

30. A. Skowroński, Generalized standard Auslander-Reiten components, J. Math. Soc. Japan 46 (1994), 517-543.

31. A. Skowroński, Group algebras of polynomial growth, Manuscr. Math. 59 (1987), 499-516.

32. A. Skowroński, Selfinjective algebras of polynomial growth, Math. Ann. 285 (1989), 177-199.

33. A. Skowroński, Cycle-finite algebras, J. Pure Appl. Algebra, 103 (1995), 105-116.

34. A. Skowroński, Module categories over tame algebras, Canadian Math. Soc. Conference Proceedings 19 (1996), 281-313.

35. A. Skowroński and $\mathrm{K}$. Yamagata, Galois coverings of selfinjective algebras by repetitive algebras, preprint, Tsukuba 1996.

Faculty of Mathematics and Informatics

Nicholas Copernicus University

ul. Chopina 12/18, 87-100 Toruń, Poland

e-mail ZYPO@MAT.UNI.TORUN.PL 